\title{
Microbiota maintain colonic homeostasis by activating TLR2/MyD88/PI3K signaling in IL-10-producing regulatory $B$ cells
}

\author{
Yoshiyuki Mishima, ${ }^{1,2}$ Akihiko Oka, ${ }^{1,2}$ Bo Liu, ${ }^{1}$ Jeremy W. Herzog, ${ }^{1}$ Chang Soo Eun, ${ }^{1,3}$ Ting-Jia Fan, ${ }^{1,4}$ Emily Bulik-Sullivan, ${ }^{5}$ \\ Ian M. Carroll, ${ }^{1,5}$ Jonathan J. Hansen, ${ }^{1,4}$ Liang Chen, ${ }^{6}$ Justin E. Wilson, ${ }^{6}$ Nancy C. Fisher, ${ }^{4}$ Jenny P.Y. Ting, ${ }^{6}$ Tomonori Nochi, ${ }^{7,8}$ \\ Angela Wahl, ${ }^{7}$ J. Victor Garcia, ${ }^{7}$ Christopher L. Karp, ${ }^{9}$ and R. Balfour Sartor ${ }^{1,4}$ \\ 'Center for Gastrointestinal Biology and Disease, Department of Medicine, Division of Gastroenterology and Hepatology, University of North Carolina at Chapel Hill (UNC), Chapel Hill, North Carolina, USA. \\ ${ }^{2}$ Department of Internal Medicine II, Shimane University Faculty of Medicine, Izumo, Shimane, Japan. ${ }^{3}$ Department of Internal Medicine, Hanyang University Guri Hospital, Guri, South Korea. ${ }^{4}$ Department of \\ Microbiology and Immunology, ${ }^{5}$ Department of Nutrition, ${ }^{6}$ Lineberger Comprehensive Cancer Center, Department of Genetics, and ${ }^{7}$ Department of Medicine, Division of Infectious Diseases, UNC, Chapel Hill, \\ North Carolina, USA. ${ }^{8}$ Mucosal Immunology Laboratory, Graduate School of Agricultural Science, Tohoku University, Sendai, Japan. ${ }^{9}$ Division of Molecular Immunology, Department of Pediatrics, Cincinnati \\ Children's Hospital Research Center, Cincinnati, Ohio, USA.
}

\begin{abstract}
Resident microbiota activates regulatory cells that modulate intestinal inflammation and promote and maintain intestinal homeostasis. IL-10 is a key mediator of immune regulatory function. Our studies describe the functional importance and mechanisms by which gut microbiota and specific microbial components influence the development of intestinal IL10-producing B cells. Using fecal transplant into germ-free (GF) I/10+/EGFP reporter and /110-/- mice, we demonstrated that microbiota from specific pathogen-free mice primarily stimulated IL-10-producing colon-specific B cells and T regulatory 1 cells in ex-CF mice. IL-10 in turn downregulated microbiota-activated mucosal inflammatory cytokines. TLR2 and -9 ligands and enteric bacterial lysates preferentially induced IL-10 production and the regulatory capacity of intestinal B cells. Analysis of I/10+/ECFP mice crossed with additional gene-deficient strains and B cell cotransfer studies demonstrated that microbiotainduced IL-10-producing intestinal B cells ameliorated chronic T cell-mediated colitis in a TLR2-, MyD88-, and PI3K-dependent fashion. In vitro studies implicated downstream signaling of PIЗKp110 $\delta$ and AKT. These studies demonstrated that resident enteric bacteria activated intestinal IL-10-producing B cells through TLR2, MyD88, and PI3K pathways. These B cells reduced colonic $\mathrm{T}$ cell activation and maintained mucosal homeostasis in response to intestinal microbiota.
\end{abstract}

\section{Introduction}

Inflammatory bowel diseases (IBDs), including Crohn's disease and ulcerative colitis, are $\mathrm{T}$ cell-mediated, chronic disorders whose development is influenced in part by dysregulated, aggressive intestinal mucosal immune reactions to resident intestinal bacteria (1-3). Enteric microbiota is essential for inducing and exacerbating chronic, immune-mediated intestinal inflammation in most rodent models of IBD. This has been most clearly demonstrated in gnotobiotic experiments in which most germ-free (GF), genetically susceptible mice and rats exhibit minimal intestinal inflammation and immune activation, but rapidly develop chronic colitis and pathogenic T cell-mediated immune responses after colonization with specific pathogen-free (SPF) or specific enteric bacteria (4-6). Recent studies have identified microbial factors that selectively stimulate pro- or antiinflammatory immune responses in the intestine. For example, segmented filamentous

Authorship note: YM and $\mathrm{AO}$ contributed equally to this work. Conflict of interest: The authors have declared that no conflict of interest exists. Copyright: $\odot$ 2019, American Society for Clinical Investigation. Submitted: March 9, 2017; Accepted: June 6, 2019; Published: August 5, 2019. Reference information: / Clin Invest. 2019;129(9):3702-3716. https://doi.org/10.1172/JC193820. bacteria strongly activate largely proinflammatory Th17 responses in the small intestine (7). On the other hand, in normal hosts, some resident enteric bacterial species stimulate regulatory immune responses that help maintain intestinal mucosal homeostasis. Selected Clostridium species induce mucosal Foxp $3^{+} \mathrm{CD} 4^{+} \mathrm{T}$ cells (8), and Bacteroides fragilis polysaccharide A stimulates IL-10-producing Tregs (9). However, these bacteria coexist in a consortium with many other species in the complex mammalian intestinal ecosystem, where a delicate balance of putative pro- and antiinflammatory species helps maintain mucosal homeostasis. In IBD, a characteristic expansion of putative aggressive bacterial groups and contraction of protective species (dysbiosis) is thought to contribute to IBD pathogenesis (1-3). Additional mechanistic studies are required in order to better understand how these resident bacterial communities interact with the host immune system to maintain intestinal homeostasis or cause IBD.

IL-10 inhibits proinflammatory, effector innate, and adaptive immune cells and helps maintain mucosal homeostasis. Mice with genetic deletion of IL-10 or the IL-10 receptor (IL-10R) spontaneously develop Th1/Th17-mediated colitis when normal enteric microbiota is present $(4,5,10)$, while administration of exogenous recombinant IL-10 prevents the onset of experimental enterocolitis, improves epithelial barrier function, and inhibits microbio- 
A
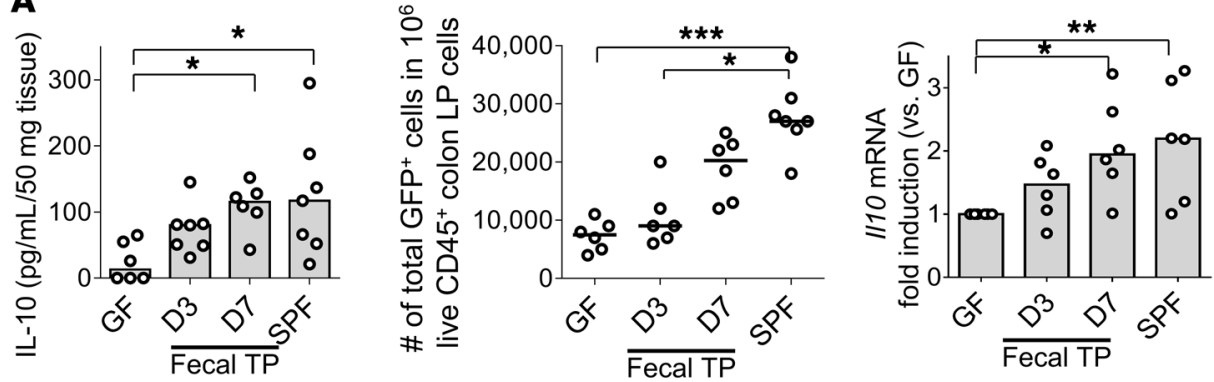

B

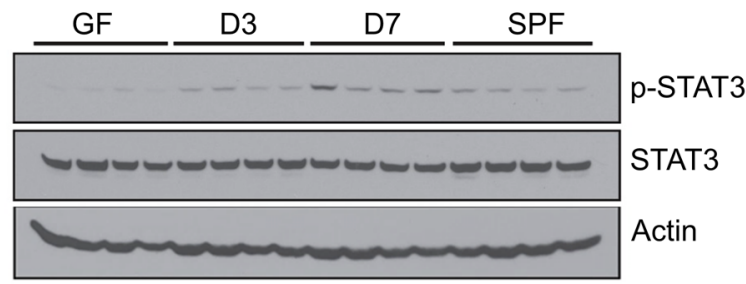

D
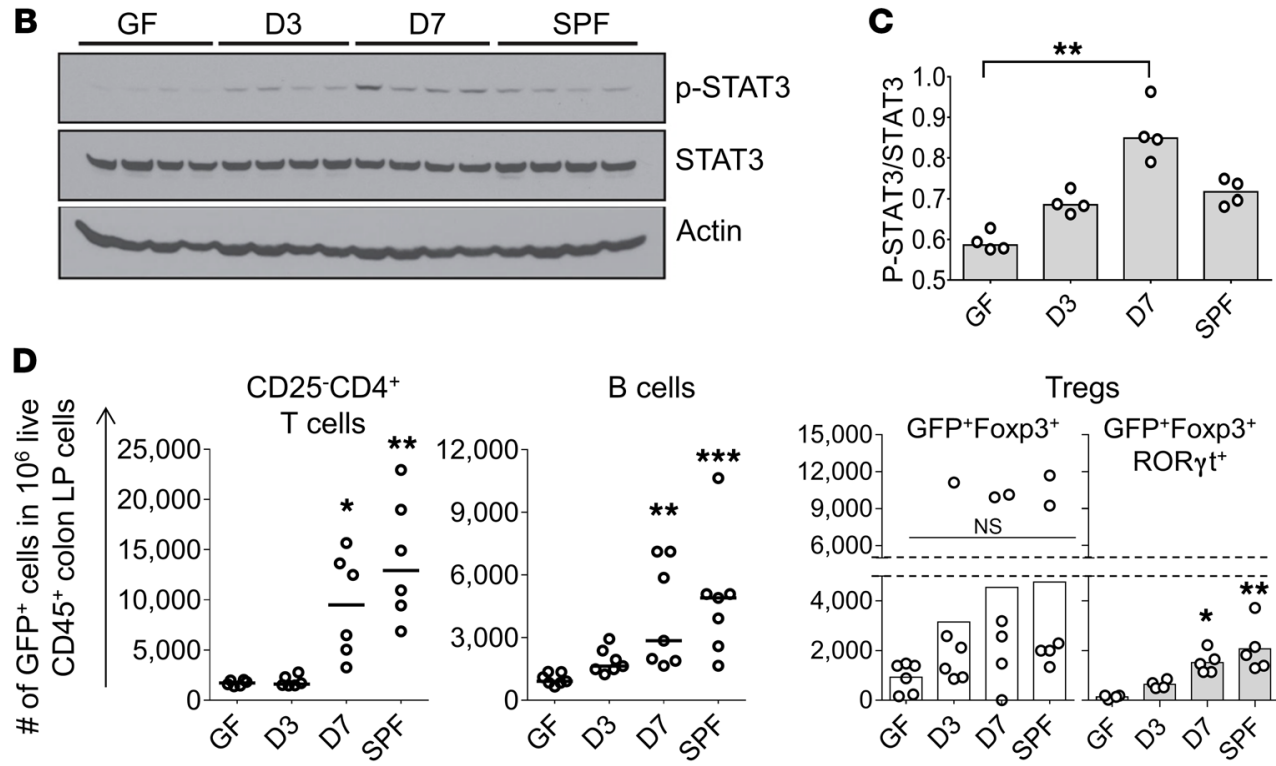

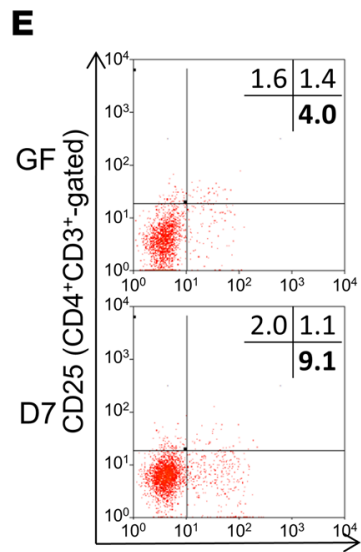

Figure 1. Resident intestinal microbiota increases the frequency of intestinal IL-10-producing immune cells and enhance IL-10 production. (A) Left: Spontaneous IL-10 secretion by colonic tissue explants; middle: number of total IL-10-producing (GFP+) colon LP cells; right: I/10 mRNA expression in dista colon tissue normalized by expression in GF mice in GF, SPF-raised, or GF-conventionalized //10+/EGFP reporter mice 3 days and 7 days (D3 and D7) after fecal transplantation (TP) with SPF feces. $n=6-9$ mice/group, combined from 2 independent experiments. (B and C) Phosphorylation levels of STAT3 in the distal colon were evaluated by Western blot analysis and quantified by densitometry. $n=4$ mice/group. (D) IL-10-producing (GFP+) colon LP cells were characterized by flow cytometry identifying cell subsets with antibodies to the indicated cell surface proteins. CD25-CD4+ ${ }^{+}$cells (CD25-CD4+CD3+), B cells

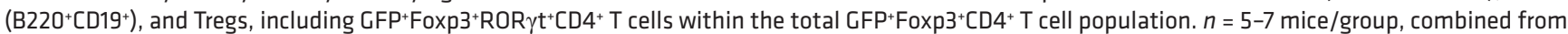

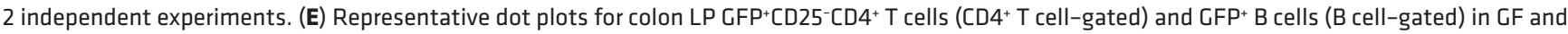
conventionalized day $7 / 110^{+/ E G F P}$ mice. For flow cytometry, the GFP+ population in live CD45 ${ }^{+}$colon LP cells was assessed using WT (GFP-) cells stained with the same antibodies as target samples as a control (see Flow cytometry in Methods). All data are presented as median values; ${ }^{*} P<0.05$, ${ }^{*} P<0.01,{ }^{*}{ }^{*} P$ $<0.001$, Kruskal-Wallis test with Dunn's post hoc test.

ta-stimulated, proinflammatory cytokine production in the mucosa $(4,11-13)$. In addition, IL-10 or IL-10R gene polymorphisms are associated with ulcerative colitis and early onset of Crohn's disease (14-16). IL-10 is produced by many types of immune cells, including $\mathrm{CD}^{+}$and $\mathrm{CD}^{+} \mathrm{T}$ cells, B cells, macrophages, DCs, neutrophils, NK cells, innate lymphoid cells, and eosinophils (17). Multiple studies implicate IL-10-producing regulatory $\mathrm{CD}^{+} \mathrm{T}$ cell subsets (Tregs, T regulatory 1 [Tr1] cells) in mucosal homeostasis $(8,18-20)$, while we and others have reported that IL-10-producing antigen-presenting cells (APCs) $(21,22)$ and regulatory B cells (23-28) are present in humans and prevent bacterial antigen-driven, $\mathrm{T}$ cell-mediated chronic experimental colitis.

Previous in vitro studies have demonstrated that TLR and Myd88 signaling contributes to the development of bacteria-induced, IL-10-secreting immune cells $(24,29,30)$. However, our current understanding of how bacterial products stimulate IL-10 production is based mainly on in vitro studies using cell lines, bone marrow-derived cells, and narrowly defined stimulants, with conflicting results in different cell types (31). Therefore, further in vivo mechanistic studies in mucosal immune cells stimulated with physiologically relevant microbial stimuli are needed to more fully understand how IL-10 mediates intestinal homeostasis.

Given our recent work demonstrating a protective role of bacteria-stimulated IL-10-secreting B cells through activation of Tregs in chronic colitis and the ability of bacterial lysates to stimulate the regulatory capacity of B cells in vitro (23), we hypothesized that TLR-dependent IL-10 production by resident bacteria is critical for B cell-mediated protection against experimental colitis. In this study, we demonstrate that resident enteric bacteria promote the in vivo development of IL-10-producing colonic lamina propria (LP) immune cells, especially T cell subsets and B cells. Intestinal bacterial colonization transiently activated proinflammatory 
A
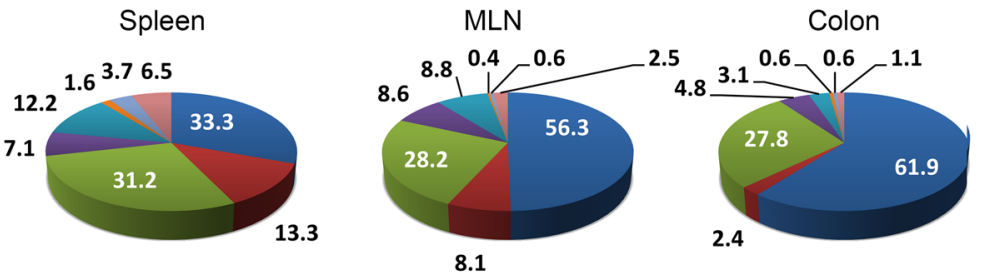
CD4 ${ }^{+} \mathrm{T}$ cells
CD8 ${ }^{+} \mathrm{T}$ cells
B cells
- DCs
Macrophages
NK cells
Granulocytes
- Plasma cells
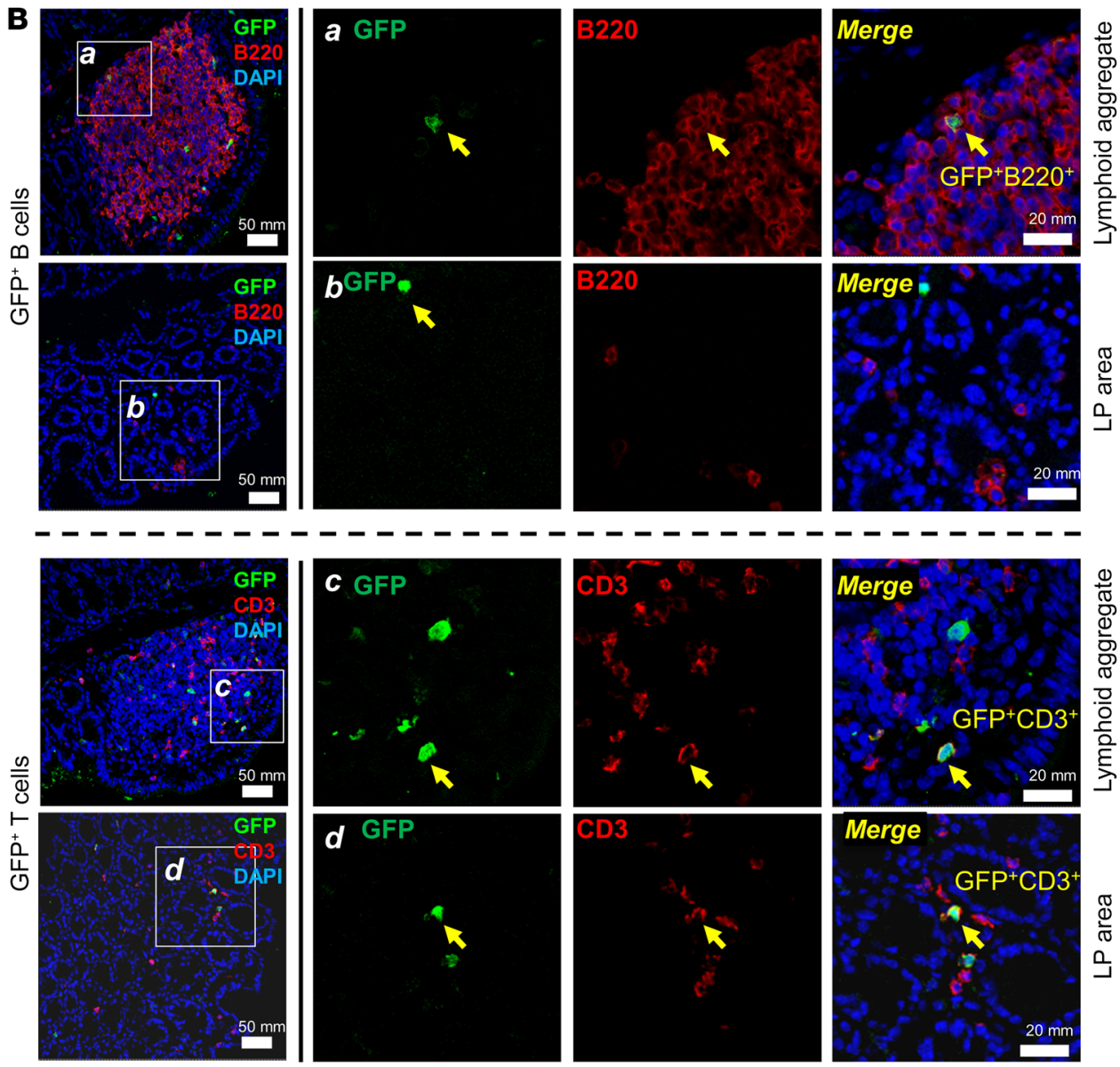

Figure 2. Distribution and characteristics of SPF mucosal and splenic GFP+ T and B cells. Physiological IL-10-producing immune cells were characterized in colonic $L P, M L N$, and spleen from SPF-raised $/ / 10^{+/ E C F P}$ reporter mice. (A) Percentages of cell types among total $\mathrm{GFP}^{+}$cells were assessed by flow cytometry using antibodies to cell surface markers as described in Methods, Flow cytometry. (B) Immunofluorescence was performed on distal colon tissue from SPF-reared $/ 110^{+/ E G F P}$ mice to characterize the distribution of GFP+ $\mathrm{B}$ cells and $\mathrm{GFP}^{+}$ $T$ cells in lymphoid aggregates and the $\mathrm{LP}$ of the distal colon. GFP+ cells are shown in green. $B$ cells $\left(B 220^{+}\right)$and $T$ cells $\left(\mathrm{CDB}^{+}\right)$are shown in red. Merged images show $B$ and $T$ cells that are GFP'. Scale bars: far left column, 50 $\mathrm{mm}$; right panels, $20 \mathrm{~mm}$. cytokines in the colon in conjunction with prolonged stimulation of IL-10 in ex-GF $I_{11 O^{+/+}}$mice, but led to sustained aggressive activation of inflammatory cytokines in the absence of IL-10. We also show that TLR2 and MyD88 signaling is essential for bacteriainducible IL-10-producing B cells to regulate $\mathrm{T}$ cell-mediated colitis and that TLR2-dependent bacterial activation of IL-10 production by B cells is mediated through the PI3K pathway. These findings increase our understanding of the pathogenesis of IBD and regulation of mucosal homeostasis by resident microbiota.

\section{Results}

Resident enteric microbiota increases intestinal IL-10-producing $T$ and $B$ cells in vivo. We used a combination of in vivo and in vitro strategies to elucidate how resident intestinal bacteria and their components stimulate protective IL-10 production by colonic LP immune cells. We employed the $I l 1 O^{+/ E G F P}$ reporter mice (32) that we derived GF (23). This model allowed us to explore the kinetics and types of IL-10-producing colonic LP cells activated by resident bacterial colonization without the ex vivo manipulation required for intracellular staining of IL-10. We first verified that $\mathrm{GFP}^{+}$cells in our $I l 10^{+/ E G F P}$ reporter mice were suitable for investigating the kinetics of IL-10-producing cells by PCR, ELISA, and flow cytometry. We found that $\mathrm{GFP}^{+} \mathrm{B}$ cells were functional, producing abundant IL-10 and lower levels of inflammatory cytokines compared with $\mathrm{GFP}^{-} \mathrm{B}$ cells following TLR ligation (Supplemental Figure 1, A-C; supplemental material available online with this article; https://doi.org/10.1172/JCI93820DS1). We developed flow cytometry methods and gating strategies to measure low-frequency $\mathrm{GFP}^{+}$cells based on basal levels of GFP fluorescence intensity in WT SPF mice and stained WT cells with the antibodies used for target samples (Supplemental Figure 1D). Our results demonstrating that the number of $\mathrm{GFP}^{+}$cells closely correlated with tissue IL-10 protein and gene expression (see below) validated the use of $\mathrm{GFP}^{+}$cells to measure physiologic IL-10 activation in vivo.

Next, to investigate whether resident bacteria induce colonic IL-10 and the kinetics of this response, we colonized GF IL-10sufficient $I l 1 O^{+/ E G F P}$ reporter mice and GF $I l 1 O^{-/-}$mice with feces 


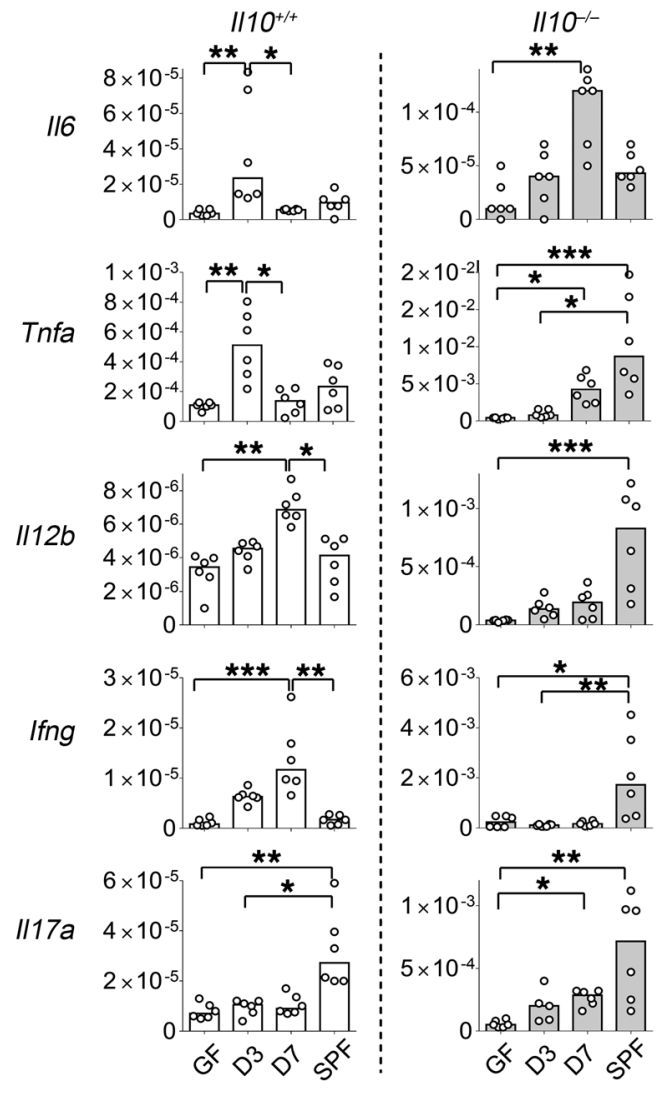

from a WT isogenic C57BL/6J mouse housed in SPF conditions and measured various immune functions 3 and 7 days later. Ageand sex-matched adult mice raised in GF or SPF conditions served as controls. We confirmed by $16 \mathrm{~S}$ rRNA assays that SPF bacteria stably colonized the intestines of ex-GF mice (Supplemental Figure 2). Spontaneous IL-10 secretion by ex vivo cultured colonic tissue explants, the frequency of $\mathrm{GFP}^{+} \mathrm{IL}-10$ reporter cells in the colonic LP, and Il10 mRNA expression in the distal colon significantly increased over time after SPF enteric bacterial colonization (Figure 1A). The frequency of $\mathrm{GFP}^{+} \mathrm{LP}$ cells strongly correlated with tissue Il10 mRNA expression (Supplemental Figure 1E) and protein secretion (Supplemental Figure 1F). In addition, SPF bacterial colonization was associated with significantly greater colonic levels of phosphorylated STAT3, a key downstream molecule in both IL-10 and IL-6 signaling (Figure 1, B and C). These results suggest that the induction of IL-10 is associated with functional signaling, though we did not investigate the relative contributions of IL-10 versus IL- 6 in activating STAT3.

We then sought to determine which subsets of colonic LP immune cells produce IL-10 in response to bacterial colonization. Bacterial colonization was associated with greater proportions of $\mathrm{GFP}^{+} \mathrm{B}$ cells and Tr1 cells (Foxp $3^{\text {neg }} \mathrm{CD} 25^{-} \mathrm{CD} 4^{+} \mathrm{T}$ cells) (Figure 1, $\mathrm{D}$ and $\mathrm{E}$, and Supplemental Figure $3 \mathrm{~A}$ ), while proportions of $\mathrm{GFP}^{+}$ macrophages, DCs, $\mathrm{CD} 8^{+} \mathrm{T}$ cells, and $\mathrm{CD} 25^{+} \mathrm{CD} 4^{+} \mathrm{T}$ cells were not significantly altered (Supplemental Figure 3B). Although the frequency of total Foxp $3^{+}$Tregs was not significantly altered by SPF fecal colonization (Supplemental Figure 3C), a newly identified Treg population (Foxp3 ${ }^{+} \mathrm{ROR} \gamma \mathrm{t}^{+} \mathrm{IL}-1 \mathrm{O}^{+} \mathrm{CD} 4^{+} \mathrm{T}$ cells) (33, 34) capable of greater immune suppression than the other Treg
Figure 3. Bacterial colonization influences the gene expression kinetics of the majority of proinflammatory cytokines in an IL-10-dependent fashion. Kinetic expression of selected genes in distal colons from GF, SPFraised (SPF), and ex-GF conventionalized ( 3 and 7 days after fecal transplantation) $/ 110^{+/ E C F P}$ reporter mice $\left(/ 110^{+/+}\right)$or $1 / 10^{-/-}$mice was determined by real-time PCR of colonic tissues. All gene expression was normalized with Actb. $n=6$ mice/group, combined from 2 independent experiments. Data are presented as median; ${ }^{*} P<0.05,{ }^{*} P<0.01,{ }^{* *} P<0.001$, Kruskal-Wallis test with Dunn's post hoc test.

types significantly increased compared with GF levels (Figure 1D, right, gray bars). These results indicate that subsets of B cells and $\mathrm{CD} 4^{+} \mathrm{T}$ cells are important sources of microbiota-activated IL-10 production in the colonic LP. Further, the proportions of $\mathrm{GFP}^{+}$ cell populations in mesenteric lymph nodes (MLNs) and spleen were not significantly altered by bacteria colonization (Supplemental Figure 3D), indicating that the observed changes were selectively induced in the colon during the experiment. These results demonstrate that bacterial colonization of GF adult mice stimulates progressively increased IL-10 production, which after 7 days approaches levels seen in SPF mice colonized at birth with resident microbiota. This IL-10 induction is slow, with significant increases in IL-10 levels compared with baseline GF levels occurring only after 7 days.

Distribution and lineage of IL-10-producing immune cells in SPF mice. We next evaluated the distribution and lineage of physiologic IL-10-producing immune cells. In mice raised in SPF conditions from birth, $\mathrm{CD} 4^{+} \mathrm{T}$ cells were the most abundant type of $\mathrm{IL}-10^{+}$cells in the colon LP (61.9\%), followed by B cells (27.8\%). B cell frequency among $\mathrm{IL}-10^{+}$cells of the colon $\mathrm{LP}$ was similar to that observed in

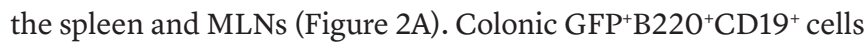
expressed $C D 5^{+} \mathrm{CD} 1 \mathrm{~d}^{\text {hi }} \mathrm{CD} 21^{\text {hi }} \mathrm{CD} 23^{-} \mathrm{CD} 24^{\text {hi }}{ }^{\mathrm{M} H C}-\mathrm{II}^{\mathrm{hi}} \mathrm{IgM}{ }^{\mathrm{lo}} \mathrm{IgD}{ }^{\mathrm{lo}}$, sharing certain phenotypic features with splenic marginal zone $\mathrm{B}$ cells and B10 cells (35) (Supplemental Figure 4). Compared with GFP- $^{-} \mathrm{B}$ cells, $\mathrm{GFP}^{+} \mathrm{LP}$ B cells had lower IgM and IgD levels and higher CD24 intensity. In mice raised in SPF conditions, IL-10producing $\mathrm{B}$ cells were located primarily within colonic mucosal lymphoid aggregates, while $\mathrm{GFP}^{+} \mathrm{T}$ cells were more diffusely distributed in both the LP and lymphoid aggregates (Figure 2B). Similarly, chronic mucosal inflammation induced by repeated dextran sulfate sodium administration for 3 cycles in the presence of resident bacteria markedly increased total mucosal IL- $10^{+}$cells (Supplemental Figure 5A), with diffuse expansion of $\mathrm{GFP}^{+} \mathrm{T}$ cells in the LP; in contrast, $\mathrm{GFP}^{+} \mathrm{B}$ cells remained limited to the lymphoid aggregates (Supplemental Figure 5, B-D), as seen in noninflamed mice (Figure 2B). Together, these results indicate that accumulation of certain subsets of IL-10-producing T and B cells in the colonic LP depends on the presence of microbiota, and these cells' prevalence increases with inflammation. This finding is consistent with previously reported induction of regulatory $\mathrm{B}$ cells by IL-1 $\beta$ and IL-6 (30).

IL-10 regulates proinflammatory genes induced by bacterial colonization. Because IL-10 regulates mucosal homeostasis, we investigated the kinetics of other inflammatory and regulatory intestinal immune responses in ex-GF mice colonized with resident enteric bacteria with and without endogenous IL-10. To do this, we examined real-time PCR expression of immune-related transcrip- 
A

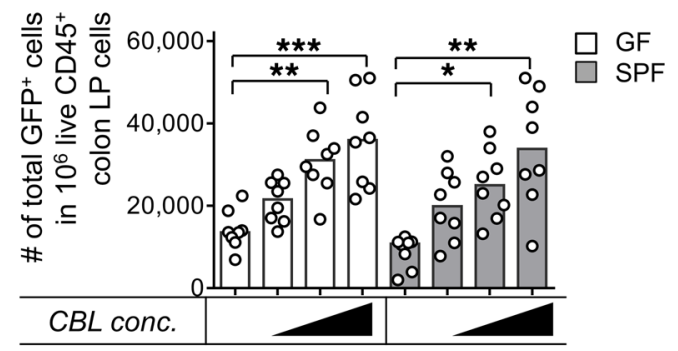

B
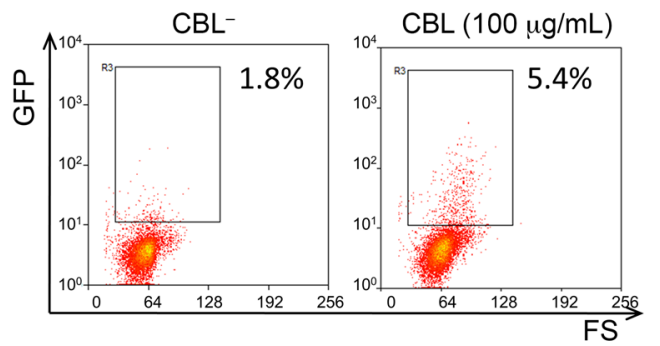

C

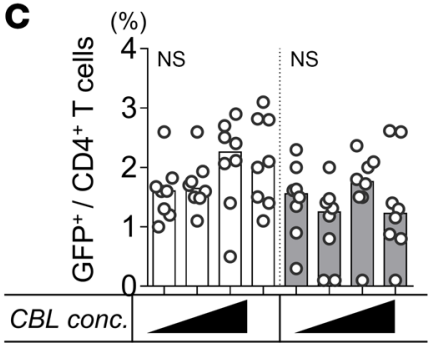

D
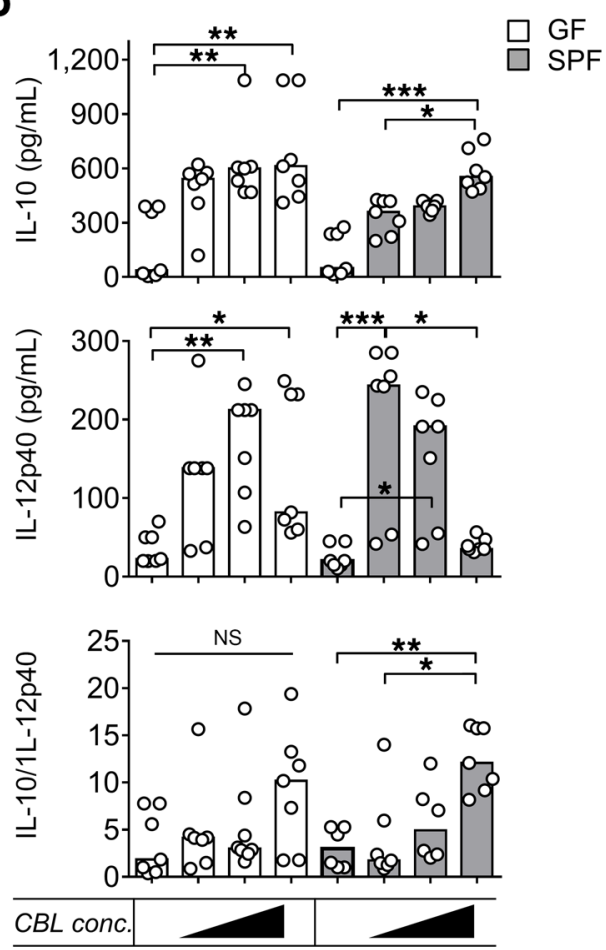

$=0,10,50,100 \mu \mathrm{g} / \mathrm{mL}$, respectively

Figure 4. CBL stimulates IL-10-producing colonic LP B cells ex vivo. Unfractionated colonic LP cells from GF or SPF-reared I/10+//EFFP reporter mice were cultured with 0-100 $\mu \mathrm{g} / \mathrm{mL}$ CBL from SPF mice for 2 days, as indicated. (A) The frequency of GFP' cells was assessed by flow cytometry. conc., concentration. (B) Representative dot plots for GFP+ cells in GF colonic cells stimulated with 0 (CBL-), 10, 50, or $100 \mu \mathrm{g} / \mathrm{mL}$ CBL. (C) Using flow cytometry, we determined frequencies of GFP-expressing $C D 4^{+} \mathrm{T}$ cells $\left(\mathrm{CD} 4^{+} \mathrm{CD} 3^{+}\right)$or $\mathrm{B}$ cells $\left(\mathrm{CD} 19^{+} \mathrm{B} 22 \mathrm{O}^{+}\right)$as a percentage of total $\mathrm{T}$ or $\mathrm{B}$ cells using control WT (GFP-) cells that stained with the same antibodies as target samples. (D) Supernatant levels of IL-10 and IL-12p40 were measured by ELISA, and IL-10/IL12 p40 ratios were calculated. Data are presented as median of 8 separated cell cultures, with cells in each culture pooled from 2-4 mice, combined from 3 independent experiments. ${ }^{*} P<0.05,{ }^{* *} P<0.01,{ }^{* *} P<0.001$, Kruskal-Wallis test with Dunn's post hoc test.

tion factors and cytokines in whole tissue from the distal colons of $\mathrm{IllO}^{+/ \mathrm{EGFP}}\left(\mathrm{IllO}^{+/+}\right)$and $\mathrm{Il10^{-/ }}$ mice reared GF, SPF-colonized ex-GF, or SPF, following the same time course used to study IL-10 induction (Figure 1A). Regulatory $I l 10$ and $T g f b 1$ were persistently upregulated in $\mathrm{IllO}^{+/+}$mice after colonization with bacteria (Figure $1 \mathrm{~A}$ and Supplemental Figure 3E). In contrast, expression of innate proinflammatory cytokine genes, including $T n f a$ and $I l 6$, was transiently upregulated 3 days after colonization (Figure 3), but downregulated by 7 days, when colonic IL-10 reached higher levels (Figure 1A). The primarily adaptive cytokine genes Ifng and $I l 12 b$ and transcription factor Tbet were continuously expressed up to 7 days after colonization, but expression of these genes was not different between GF and SPF mice (Figure 3 and Supplemental Figure 3E), suggesting that either more prolonged stimulation after bacterial exposure or early-life exposure to microbiota is required in order to downregulate these cytokines. In contrast, Il17a expression did not increase following bacterial colonization of GF mice, but was higher in mice born SPF than in GF mice. Rorgt was persistently upregulated in $\mathrm{IllO}^{+/+}$mice (Supplemental Figure 3E), which might be associated with induction of Foxp $3^{+} \mathrm{ROR} \gamma \mathrm{t}^{+} \mathrm{IL}-1 \mathrm{O}^{+}$Tregs (Figure 1D, right). Moreover, whole colonic tissue Foxp 3 mRNA expression was not significantly altered by bacterial colonization, consistent with the lack of significant changes in the frequency of total LP Foxp $^{+} \mathrm{T}$ cells in GF, ex-GF-colonized, and SPF mice (Figure 1D and Supplemental Figure 3, C and E). To determine the role of endogenous IL-10 in regulating these proinflammatory genes, we preformed similar studies in $\mathrm{Il}_{1} \mathrm{O}^{--}$mice. In contrast to the transient increase in inflammatory genes in WT mice, colonization of $\mathrm{Il}_{10} \mathrm{O}^{--}$mice with identical SPF fecal microbiota was associated with a far more robust and persistent increase in proinflammatory cytokine gene expression in the distal colon, with levels 1- to 2-log higher than in $I l 1 \mathrm{O}^{+/+}$mice (Figure 3). We had previously shown similar bacterial composition up to 1 week after fecal transplantation in WT and $I l 10^{-/-}$mice (36). Tgfbl was also continuously upregulated in $\mathrm{Il}_{10 /-}$ mice (data not shown), suggesting that transient upregulation of proinflammatory cytokine genes with subsequent suppression in $\mathrm{IlOO}^{+/+}$animals was either mainly IL-10 dependent or that an antiinflammatory effect of TGF- $\beta 1$ was negated in the absence of IL-10. These results suggest that resident bacteriainduced IL-10 helps maintain intestinal homeostasis by downregulating aggressive proinflammatory responses induced by resident microbiota. This conclusion is consistent with our previous observation that in vivo neutralization of IL-10R increases IFN- $\gamma$ responses to resident bacterial antigens (13). 


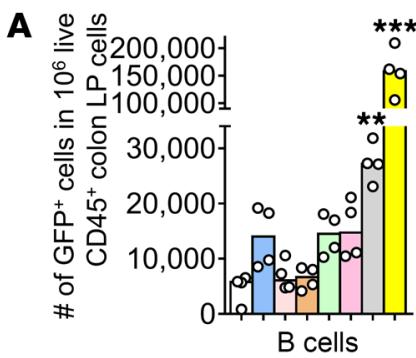

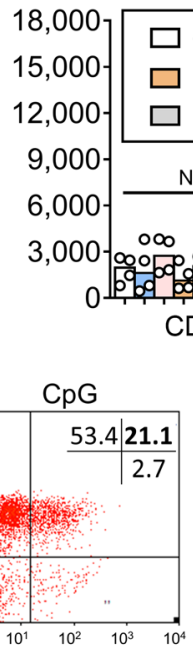
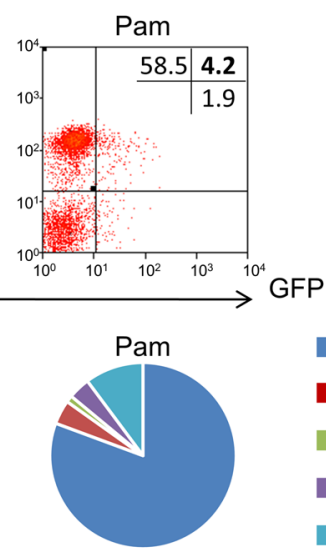

B cells

CD4 ${ }^{+} \mathrm{T}$ cells

CD8 ${ }^{+} \mathrm{T}$ cells

DCs

Macrophages
Figure 5. Bacterial products predominantly expand $\mathrm{GFP}^{+} B$ cells and innate immune cells ex vivo. Unfractionated colonic LP cells from GF $1 / 10^{+/ E C F P}$ reporter mice were cultured without (-, media only) or with $200 \mathrm{ng} / \mathrm{mL}$ LPS, 50 ng/mL Pam3csk (Pam), 1 nM CpGDNA (CpG), $10 \mu \mathrm{g} / \mathrm{mL}$ lysates of E. coli LF82 $(\mathrm{Ec}), E$. faecalis $(\mathrm{Ef})$, or R. gnavus $(\mathrm{Rg})$ or a mixture of 17 strains of Clostridia species (Clo). (A) Frequencies of GFP-expressing cell types were determined by flow cytometry using antibodies to cell surface markers as described in Methods. Data are presented as median of 4 separate cell cultures, with cells in each culture pooled from 2-4 mice; ${ }^{*} P<0.05$, ${ }^{* *} P<0.01,{ }^{* * *} P<0.001$ (vs. no stimulation), Kruskal-Wallis test with Dunn's post hoc test. (B) Representative dot plots for GFP (IL-10)+ B cells stimulated with or without $\mathrm{CpG}$ or Pam3. (C) Summary pie charts for cell populations expressing GFP.
Luminal bacteria increase the regulatory capacity of intestinal IL-1O-secreting B cellsexvivo. Having established that resident microbiota induces in vivo IL-10 expression in the colon and that endogenous IL-10 inhibits proinflammatory responses, we next characterized the IL-10-secreting immune cell types that were activated following ex vivo cecal bacterial lysate (CBL) treatment of cultured, unfractionated colon LP cells from GF versus SPF Il10+/EGFP mice. We have used CBL from SPF WT mice to replicate physiologic bacterial stimulation in previous ex vivo cellular activation studies $(5,21,23)$. In the current experiments, ex vivo CBL stimulation significantly increased the percentage of $\mathrm{GFP}^{+}$immune cells in a dose-dependent manner regardless of the source of cells (GF vs. SPF) (Figure 4, A and B). We observed a dose-dependent increase in numbers of $\mathrm{GFP}^{+} \mathrm{B}$ cells, but not $\mathrm{CD} 4^{+} \mathrm{T}$ cells, following $\mathrm{CBL}$ stimulation (Figure 4C). IL-10 protein levels also increased in response to CBL stimulation, although the GF LP cells appeared to be more sensitive to low concentrations of CBL than did SPF cells, so that a dose-dependent response was not seen in GF cells. IL-10 protein secretion correlated strongly with the frequency of $\mathrm{GFP}^{+} \mathrm{B}$ cells $\left(r^{2}=0.766, P<0.0001\right)$. Levels of IL-12p40, a representative inflammatory cytokine that induces Th1/Th17 immune responses, in cells derived from SPF mice steadily decreased as CBL concentrations increased, but decreased only with the highest dose of CBL in GF cells. These results indicate a bacteria-mediated regulatory phenotype with elevated IL-10/IL-12p40 ratios in response to a high dose of CBL (Figure 4D).

To examine the role of endogenous IL-10 in regulating in vitro induction of proinflammatory IL-12 by intestinal bacterial stimulation, we performed similar studies in cells from IL-10-deficient mice and in WT IL-10-sufficient mouse cells treated with neutralizing IL-10R to block IL-10 signaling. High-dose bacterial lysate-induced downregulation of IL-12p40 was not seen in LP cells from SPF $\mathrm{Il1O}^{-/}$mice (Supplemental Figure 6A), and levels of IL-12p40 in these mice were 2- to 3-fold greater than those found in WT cells stimulated by the lower doses of CBL (Figure 4D). These contrasting results in WT and $I l 10^{-/-}$cells suggest that IL-10 plays an important role in inducing the regulatory capacity of LP cells. This conclusion was confirmed by the observation that antiIL-10R antibody administration significantly increased IL-12p40 production by WT LP cells stimulated ex vivo with high-dose CBL (Supplemental Figure 6B).

Bacterial TLR ligands selectively induce $I L-10$ production by $L P$ innate immune cells. Having established that unfractionated bacterial microbiota induces IL-10 production by $\mathrm{LP} \mathrm{CD} 4^{+} \mathrm{T}$ cells and $B$ cells, we next sought to identify the bacterial components that induce IL-10 production by colonic LP cells. We performed in vitro stimulation using representative TLR ligands and lysates of representative classes of resident bacteria implicated in IBD pathogenesis and mucosal protection. We stimulated colonic LP cells from GF mice with (i) the TLR ligands Pam3csk4 (Pam3: TLR $1 / 2$ agonist), LPS (TLR4 agonist), and CpG-DNA (TLR9 ligand); (ii) lysates from putative proinflammatory human bacterial species Escherichia coli LF82, Enterococcus faecalis, and Ruminococcus gnavus (more prevalent in patients with active IBD and inducers of experimental colitis in gnotobiotic IL-10-deficient mice; refs. 2,36 ); and (iii) a mixture of 17 antiinflammatory strains of human Clostridium species that protect against experimental colitis (8) and are decreased in IBD patients $(3,37)$. We detected increased frequencies of $\mathrm{GFP}^{+}$colon LP B cells in cultures that were treated with Pam3 or CpG-DNA (Figure 5, A and B). Frequencies of GFP ${ }^{+}$ macrophages and DCs also increased following ex vivo stimulation with CPG-DNA, but the number of these cells was lower than that of IL-10-producing B cells by 10 - to 25 -fold (Figure 5C). These results are consistent with a higher level of secreted IL-10 
A
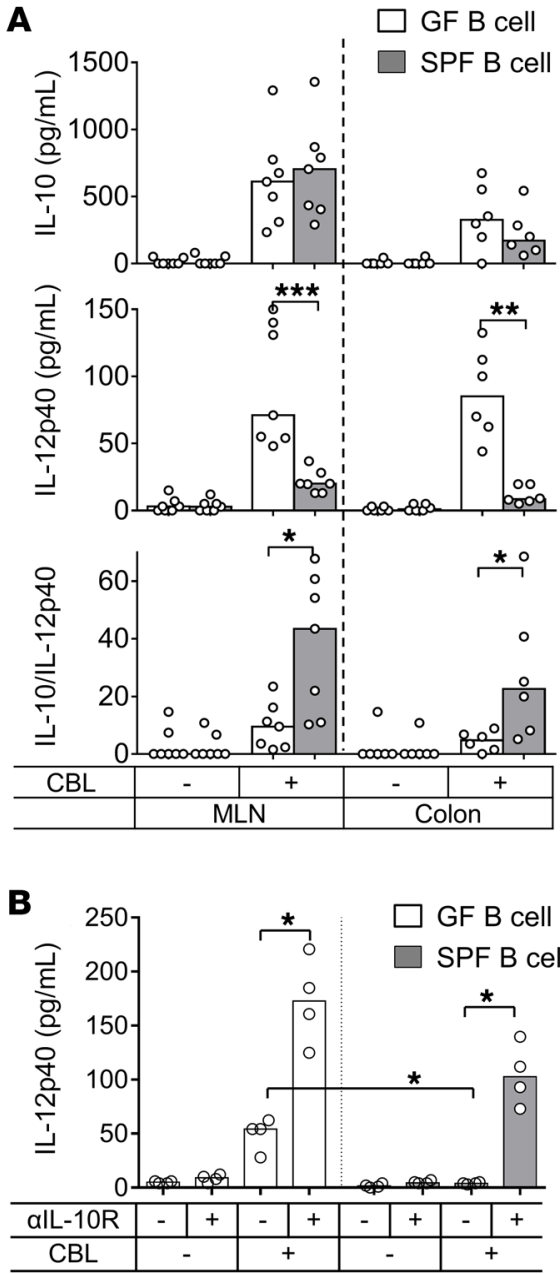

C

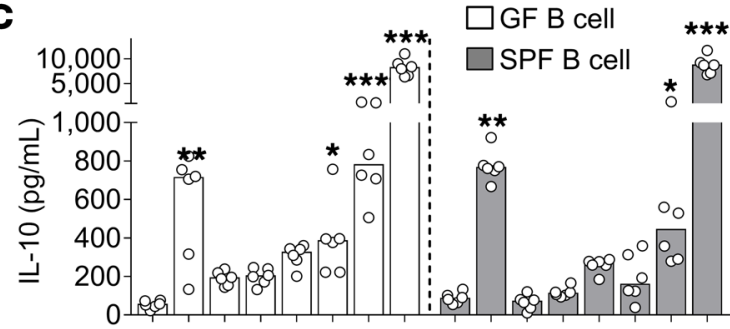

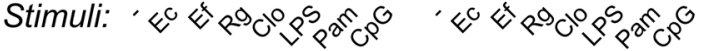

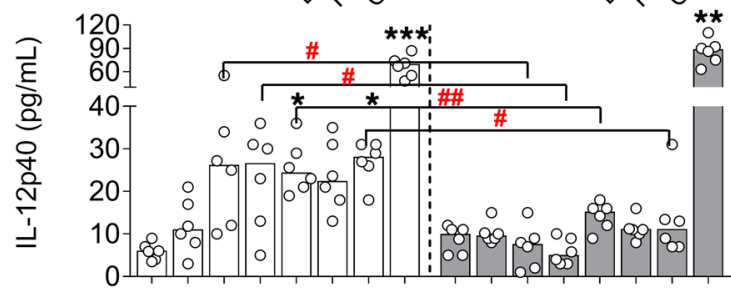

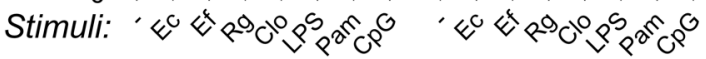

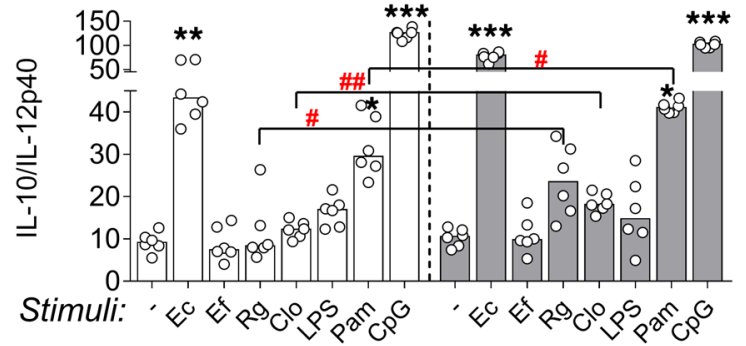

Figure 6. Enteric resident bacteria in mice increase the capacity of intestinal B cells to suppress ex vivo bacterial lysate-stimulated inflammatory cytokine production that is mediated by IL-10 signaling. (A) Cytokine secretion by $10 \mu \mathrm{g} / \mathrm{mL}$ CBL-stimulated colonic LP B cells isolated from GF or SPFraised WT mice in culture for 2 days. Cytokine concentrations were measured by ELISA. Data are presented as median of 6-7 separate cell cultures, with cells in each culture pooled from 3-4 mice. Mann-Whitney $U$ test (GF vs. SPF). ${ }^{*} P<0.05,{ }^{* *} P<0.01,{ }^{* *} P<0.001$. (B) Colonic B cells from GF or SPF-reared WT mice were cultured with anti-IL-10R or isotype control antibodies in the presence or absence of $10 \mu \mathrm{g} / \mathrm{mL}$ CBL for 24 hours. Supernatant levels of IL-12p40 were measured by ELISA. Data are presented as median of 4 separate cell cultures, with cells in each culture pooled from 2-4 mice; ${ }^{*} P<0.05$, Kruskal-Wallis test with Dunn's post hoc test. (C) Cytokine secretion by MLN B cells from GF or SPF-raised WT mice cultured for 2 days with the indicated bacteria lysates or TLR ligands (see Figure 5 legend). IL-10 and IL-12p40 in culture supernatants were measured by ELISA. Data are presented as median of 4 separate cell cultures, with cells in each culture pooled from 3-6 mice. ${ }^{*} P<0.05,{ }^{*} P<0.01,{ }^{* * *} P<0.001$ (vs. no stimulation), Kruskal-Wallis test with Dunn's post hoc test; ${ }^{\#}<0.05,{ }^{\#} P<0.01$ (GF vs. SPF), Mann-Whitney $U$ test.

following ex vivo stimulation of colonic LP cells with Pam3 and CPG (Supplemental Figure 6C). The frequencies of $\mathrm{GFP}^{+} \mathrm{CD}^{+}$ $\mathrm{T}$ cells were not significantly altered by any of the in vitro stimuli tested (Figure 5A). These data indicate that ex vivo stimulation with bacterial products preferentially induces increased numbers of immunoregulatory IL-10-producing B cells compared with $\mathrm{CD} 4^{+} \mathrm{T}$ cells. $\mathrm{CD} 4^{+} \mathrm{T}$ cells may require additional signals, longer activation times, or specific antigenic stimulation of interacting APCs that are more efficient in vivo to produce IL-10.

In vivo exposure to resident microbiota programs protective $B$ cell responses to ex vivo bacterial stimulation of $I L-10$ and inflammatory cytokine production. Given that treatment with CBLs and selected TLR ligands increased the frequency of IL-10-producing B cells and IL-10 production in ex vivo cultures of LP cells isolated from GF mice, we next examined (i) whether in vivo exposure to resident microbiota influences innate immune responses to ex vivo bacterial products and (ii) whether these products differentially activate protective IL-10 and a key proinflammatory cytokine, IL-12p40. We stimulated colonic LP B cells from GF and SPF mice first with a physiologic colonic microbiota lysate, CBL, then with representative bacterial lysates and TLR ligands. We measured the relative induction of IL-10 and IL-12p40 secretion by ELISA. CBL-stimulated intestinal (MLN and colon LP) B cells produced abundant IL-10 ex vivo regardless of the source of cells (GF vs. SPF) (Figure 6A). However, IL-12p40 production by CBL-stimulated intestinal B cells from SPF mice was significantly lower than from B cells derived from GF mice, resulting in a more protective phenotype, as indicated by increased IL-10/IL-12p40 ratios in CBL-stimulated SPF B cells (Figure 6A). Blockade of IL-10 signaling with anti-IL-10R antibody increased IL-12p40 production in both SPF and GF colonic B cells, 
A

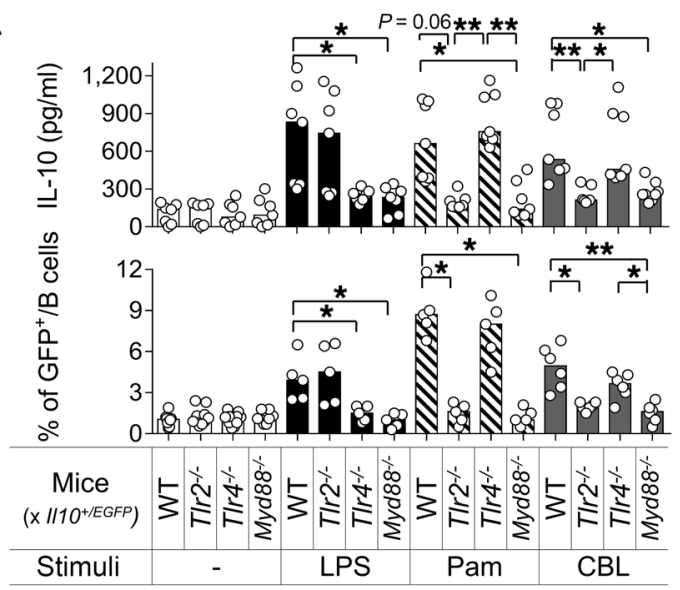

C

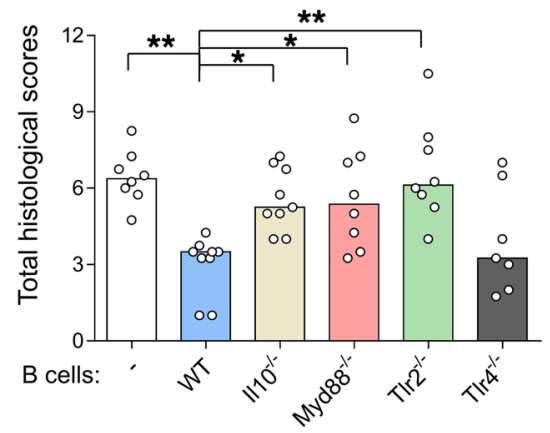

D

B
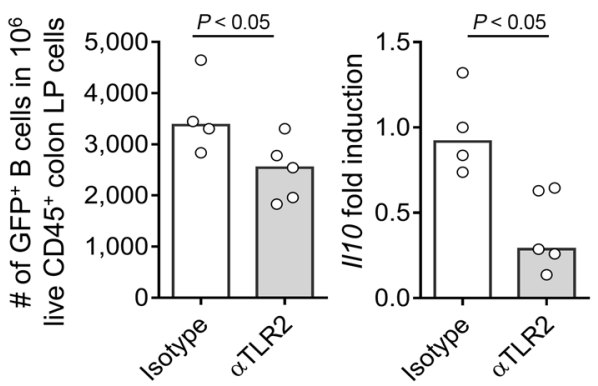

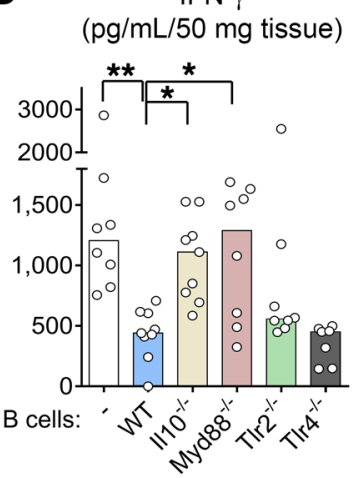

$\mathrm{IL}-10$

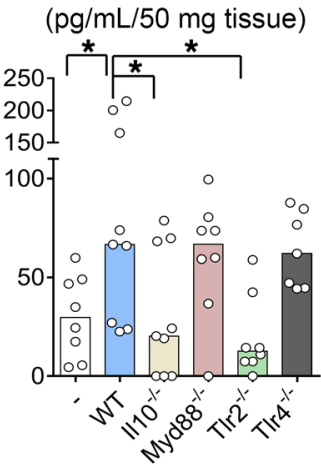

IL-10/IFN- $\gamma$ ratio

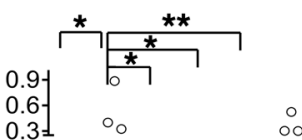

00

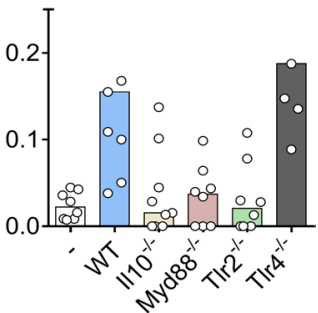

Figure 7. TLR2/MyD88 signaling increases the frequency of IL-10-producing B cells upon bacterial product stimulation ex vivo and mediates the suppression

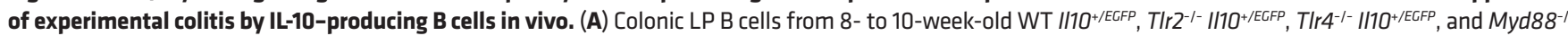
$\| 110^{+/ E C F P}$ mice were cultured without (-) or with $10 \mu \mathrm{g} / \mathrm{mL}$ CBL, $50 \mathrm{ng} / \mathrm{mL}$ Pam3, or $200 \mathrm{ng} / \mathrm{mL}$ LPS for 24 hours, after which IL-10 levels in culture supernatants were measured by ELISA (top panel). GFP' populations in B cells (live CD45+CD19+B220+) were analyzed with flow cytometry in reference to WT (GFP-) control cells stained with the same antibodies as target samples. Data are presented as median of 7-8 separate cell cultures, with cells in each culture pooled from 2-3 mice, combined from 3 independent experiments (bottom panel). ${ }^{*} P<0.05,{ }^{*} P<0.01$. (B) Eight-week-old GF $/ 110^{+/ E C F P}$ reporter mice were conventionalized with SPF fecal bacterial transplantation. Mice were given anti-TLR2 antibody ( $\alpha$ TLR2) or isotype control i.v. on days 0 and 4 after fecal bacterial transplantation and harvested on day 7 for IL-10 analysis. Colonic LP cells were isolated, and live CD45+CFP+ B cells (CD19+B220+) were analyzed by flow cytometry (left) as described above; II10 mRNA levels were normalized with Actb (right). $n=4-5$ mice. Data are presented as median, Mann-Whitney $U$ test. (C and D) Splenic B cells ( $\left.1 \times 10^{6}\right)$

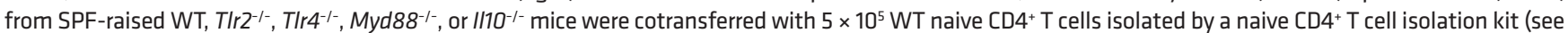

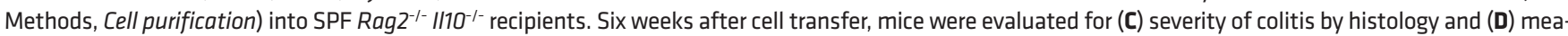
surement of spontaneously secreted IL-10 and IFN- $\gamma$ in colonic tissue explants by ELISA. $n=7-9$ mice/group, combined from 2 independent experiments. Data are presented as median; ${ }^{*} P<0.05$ and ${ }^{*} P<0.01$ (vs. WT mice), Kruskal-Wallis test with Dunn's post hoc test.

indicating that IL-10 signaling is essential for inducing a regulatory phenotype in mucosal B cells (Figure 6B). To assess the functional significance of physiologic bacterial lysate activation of regulatory $\mathrm{B}$ cells, we created B cell/T cell $\left(\mathrm{CD}^{+}\right)$cocultures with and without CBL stimulation and measured $\mathrm{T}$ cell proliferation and effector cytokine production. Colonic B cells from SPF WT mice suppressed $\mathrm{T}$ cell proliferation and IFN- $\gamma$ and IL-17a production in a B cell number-dependent manner with, but not without, CBL stimulation (Supplemental Figure 7, A-C). These results illustrate the importance of resident enteric microbiota and IL-10 signaling for the healthy development and function of intestinal regulatory B cells.

To identify the specific bacterial species and components that stimulate B cells to secrete IL-10, we treated MLN B cells from GF versus SPF mice with the spectrum of bacterial species lysates and TLR ligands described in the experiments shown in Figure 5. Both GF and SPF MLN B cells secreted abundant IL-10 in response to
Pam3, CpG-DNA, and E. coli LF82 lysate (Figure 6C). However, while secretion of IL-10 by stimulated MLN B cells was similar in GF and SPF mice, MLN B cells from SPF-raised animals produced less IL-12p40 compared with GF mice when treated with lysates of E. faecalis, R. gnavus, and Clostridium species, and with Pam3 but not CpG-DNA. IL-10/IL-12p40 ratios were also higher in cultures of cells from SPF versus GF mice for R. gnavus, Clostridia, and Pam3 stimuli (Figure 6C). These observations are similar to those in CBL-stimulated MLN B cells (Figure 6A). In aggregate, these results demonstrate that intestinal B cells acquire a strong regulatory (tolerogenic) phenotype following in vivo exposure to complex resident bacteria that is maintained by continuous ex vivo stimulation with TLR2 and -9 ligands.

IL-10-secreting B cells are induced and inhibit colitis in a TLR2and MyD88-dependent manner. Based on our observations that in vitro exposure of resident microbiota and bacterial TLR ligands 
A

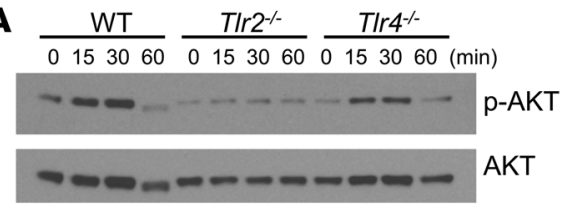

B
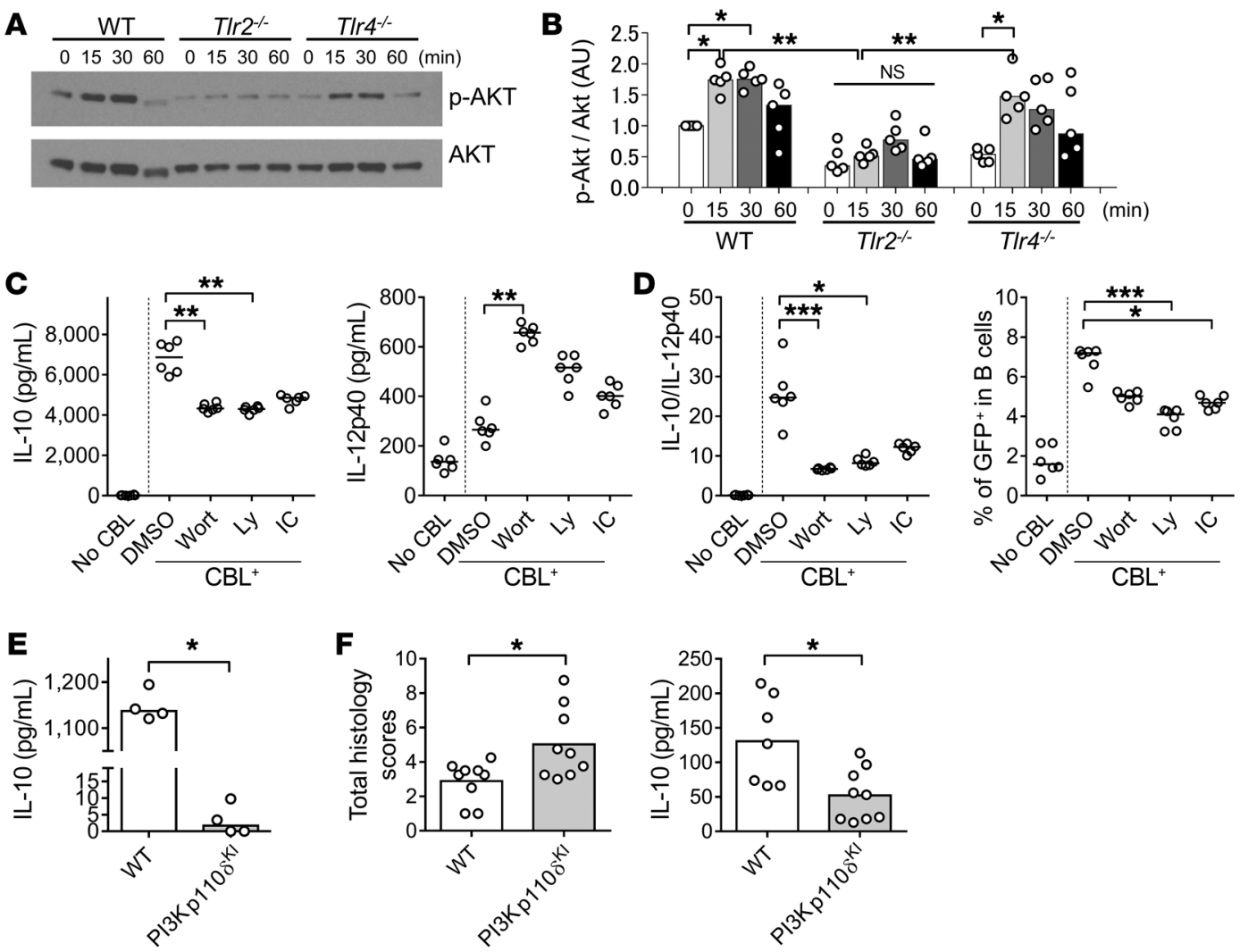

Figure 8. The PI3K/AKT pathway is involved in TLR2-dependent IL-10 production by CBL-stimulated B cells and their regulatory effect on T cell-mediated colitis. Splenic B cells $\left(1 \times 10^{6} /\right.$ well) from 8 - to 10 -week-old SPF WT, T/r2 ${ }^{-1-}$, and T/r $4^{-1-}$ mice were cultured without or with $10 \mu \mathrm{g} / \mathrm{mL}$ CBL. Cells were harvested $0,15,30$, and 60 minutes after CBL stimulation, and phosphorylation of AKT was analyzed by Western blot. (A) Representative blots and (B) densitometric analysis of phospho-AKT/AKT are shown. $n=5$ mice/group, combined from 2 independent experiments. ${ }^{*} P<0.05,{ }^{*} P<0.01$, Kruskal-Wallis test with Dunn's post hoc test. (C) Splenic B cells $\left(1 \times 10^{6} /\right.$ well) from 8 - to 10 -week-old SPF $/ 110^{+/ E C F P}$ mice were cultured with $1 \mu \mathrm{M}$ pan-PI3K inhibitor wortmannin (Wort) or Ly294002 (Ly), $1 \mu$ M PI3Kp110 $\delta$-specific inhibitor IC-87114 (IC), or DMSO alone, with stimulation with $10 \mu \mathrm{g} / \mathrm{mL}$ CBL for 48 hours. Supernatant levels of IL-10 and IL-12p40 were measured by ELISA. (D) GFP+ population in B cells (live CD45+CD19+B220+) were analyzed by flow cytometry in reference to WT $\left(\mathrm{GFP}^{-}\right)$control cells stained with the same antibodies as target samples. Data are presented as median of 6 separate cell cultures, combined from 2 independent experiments. ${ }^{*} P<0.05,{ }^{* *} P<0.01,{ }^{* *} P<0.001$, Kruskal-Wallis test with Dunn's post hoc test. (E) Splenic B cells $\left(5 \times 10^{5} /\right.$ well) from 8- to 10 -week-old SPF WT and PI3Kp $110 \delta^{\mathrm{KI}}$ mice were cultured with $10 \mu \mathrm{g} / \mathrm{mL}$ CBL for 48 hours. Supernatant levels of IL-10 were measured by ELISA. Data are presented as median. $n=4$ mice, combined from 2 independent experiments. ${ }^{*} P<0.05$, Mann-Whitney $U$ test. (F) Splenic B cells $\left(1 \times 10^{6}\right)$ from SPF-raised WT or PI3K-

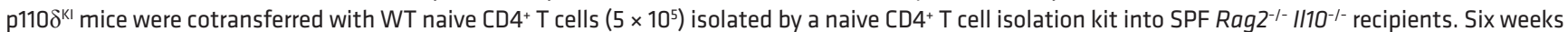
after cell transfer, mice were evaluated for severity of colitis by histology and measurement of spontaneously secreted IL-10 levels in colonic tissue explants by ELISA. $n=7-9$ mice/ group, combined from 2 independent experiments. Data are presented as median; ${ }^{*} P<0.05$, Mann-Whitney $U$ test.

induces IL-10-secreting mucosal cells, including B cells with a regulatory phenotype, we next sought to determine (i) whether TLR signaling helps develop physiological or bacteria-induced IL-10-secreting mucosal cells; and (ii) whether this pathway mediates mucosal protection by regulatory B cells. Based on our observations that a TLR2 ligand consistently activated IL-10 production, we created SPF $I l 10^{+/ E G F P} \operatorname{Tl} 2^{-/}$, Ill ${ }^{+/ E G F P} \mathrm{Tlr} 4^{-/}$, and $I l 10^{+/ E G F P}$ Myd $88^{-/}$double mutants to enable in vivo and ex vivo functional studies. We used TLR4 as a thoroughly investigated parallel pathway for LPS- and Gram-negative bacteria-induced activation and MyD88 as a common TLR2 and TLR4 downstream signaling molecule. We first quantified IL-10-secreting cells in the colon LP of these mice in vivo and after ex vivo stimulation with the TLR ligands LPS, Pam3, and CBL. We found that the absence of TLR2, TLR4, or MyD88 signaling did not significantly alter either the percentage of colonic LP $\mathrm{GFP}^{+} \mathrm{B}$ cells or basal secre- tion of IL-10 by cultured LP B cells without ex vivo stimulation (Figure 7A and Supplemental Figure 8A). However, ex vivo CBL stimulation of cultured colonic LP cells significantly increased IL-10 secretion and the proportion of $\mathrm{GFP}^{+} \mathrm{B}$ cells in a TLR2/ MyD88-dependent, but TLR4-independent, manner (Figure 7A and Supplemental Figure 8A). As expected, LPS and Pam3 stimulation of cultured colonic LP cells increased IL-10 secretion and the proportion of $\mathrm{GFP}^{+} \mathrm{B}$ cells in a TLR4/MyD88-dependent and TLR2/MyD88-dependent manner, respectively (Figure 7A). As in other experiments, the number of $\mathrm{GFP}^{+} \mathrm{B}$ cells strongly correlated with IL-10 protein production $\left(r^{2}=0.724, P<0.0001\right)$. We then verified that $B$ cells are a primary source of IL-10 production following ex vivo intestinal bacterial stimulation in the absence of other cell populations (Supplemental Figure 9). These data sug-

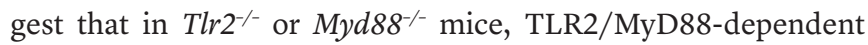
$\mathrm{IL}-10$ production by $\mathrm{B}$ cells is not due to abnormal immune activa- 
tion by other cell types, for example, aberrant T cell activation. On the other hand, CBL-stimulated IL-12p40 production by unfractionated colonic LP cells was not altered by lack of TLR signaling (Supplemental Figure 8B), likely due to predominant IL-12p40 production by non-B cell populations (i.e., macrophages and DCs) by other stimulatory pathways. In aggregate, these results demonstrate that global resident bacterial induction of IL-10-secreting B cells ex vivo is TLR2 and MyD88 dependent. In addition, in vivo blockade of TLR2 by anti-TLR2 antibody in GF 1 l10 ${ }^{+/ E G F P}$ reporter mice during colonization with SPF bacteria resulted in diminished numbers of colonic $\mathrm{GFP}^{+} \mathrm{B}$ cells and decreased colonic $I l 10$ mRNA expression (Figure 7B). These results emphasize the importance of TLR2 signaling in enteric bacteria-mediated, intestinal IL-10-producing B cell development and activation.

Based on these results showing TLR2/MyD88-dependent induction of IL-10-producing B cells in vivo and in vitro, we examined the regulatory function of bacteria-induced IL-10-secreting $\mathrm{B}$ cells in vivo using our established $\mathrm{CD} 4^{+} \mathrm{T}$ and $\mathrm{B}$ cell cotransfer colitis model (23). We cotransferred splenic naive $\mathrm{CD}^{+}{ }^{+} \mathrm{T}$ cells from WT mice with or without splenic B cells isolated from SPFraised WT, $\mathrm{Tlr}^{-/-}, \mathrm{Tlr}^{-/-}, \mathrm{IllO}^{-/-}$, and $\mathrm{Myd88^{-/ }}$ mice into $\mathrm{IllO}^{-/-}$ $\operatorname{Rag}^{-1-}$ mice, and evaluated the severity of colitis 6 weeks after cell transfer. Transferred WT and $\mathrm{Tlr}^{-/-}$B cells substantially suppressed histological evidence of colonic inflammation (Figure 7C and Supplemental Figure 10) and were associated with lower spontaneous IFN- $\gamma$ secretion, higher spontaneous IL-10 secretion, and elevated IL-10/IFN- $\gamma$ ratios by colonic explants (Figure 7D). On the other hand, B cells lacking IL-10, TLR2, or MyD88 exhibited no protective function (Figure 7, C and D, and Supplemental Figure 10), although transferred Tlr $2^{-/-}$B cells were present in the colon (data not shown). Based on these results in the T and B cell cotransfer colitis model, we propose that mucosal IL-10-secreting $\mathrm{B}$ cells protect against chronic colitis in a microbiota-dependent manner through selective TLR signaling.

TLR2-specific CBL activation in B cells activates the PI3K/AKT/ glycogen synthase kinase $3 \beta$ signaling pathway. Since colonization with resident microbiota or ex vivo stimulation with CBL induces IL-10 production by colonic B cells with a regulatory phenotype in a TLR2-dependent fashion, we next explored downstream signaling pathways by measuring activation of signaling components by Western blotting and selective pharmacologic and genetic blockade of key signaling molecules. We first investigated the TLR2-specific activation of the PI3K/AKT pathway, which has been found to facilitate IL-10 production $(31,38,39)$. We cultured colonic LP B cells from SPF WT, Tlr2 ${ }^{-/}$, and $\mathrm{Tlr} 4^{-/-}$mice with CBL, and measured phosphorylation of AKT, a key downstream molecule in thePI3K pathway. We found that phosphorylation of AKT was activated to a significantly lesser extent by CBL in Tlr2 $2^{-/}$B cells compared with WT and Tlr4 $4^{-/}$B cells (Figure $8, \mathrm{~A}$ and $\mathrm{B}$ ). We also observed that the PI3K pathway-related molecules PDK and glycogen synthase kinase $3 \beta$ (GSK3 $\beta$ ), but not $\mathrm{p} 85$, were activated in a TLR2-dependent manner, while the Myd88 signaling-associated molecules p38 and ERK were not dependent on TLR2 activation (Supplemental Figure 11, A and B). These results indicate that CBL stimulation preferentially activates the PI3K/AKT/GSK3 $\beta$ signaling pathway downstream of TLR2 in B cells. To confirm these findings functionally, we performed targeted inhibitor studies using CBL-stimulated B cell production of IL-10 and IL-12p40 (Figure $8 \mathrm{C}$ ). We found that PI3K, including the PI3Kp110 $\delta$ subunit, was involved in CBL-stimulated IL-10 production by B cells and regulatory function characterized by an increased IL-10/IL-12p40 ratio (Figure 8C). Increased CBL-stimulated $\mathrm{GFP}^{+} \mathrm{B}$ cell frequency was also inhibited by pan-PI3K and PI3Kp110 $\delta$-specific inhibitors (Figure $8 \mathrm{D}$ and Supplemental Figure 12). PI3K110 $\delta^{\mathrm{KI}} \mathrm{B}$ cells were neither able to produce abundant IL-10 following in vitro CBL stimulation (Figure 8E) nor ameliorate $\mathrm{T}$ cell-mediated colitis in the $\mathrm{CD}^{+} \mathrm{T}$ and $\mathrm{B}$ cell cotransfer model (Figure $8 \mathrm{~F}$ ). Together, our in vivo and in vitro studies indicate that protective IL-10 production by $\mathrm{B}$ cells is activated by CBL through TLR2 and downstream signaling through the PI3K (p110 $\delta$ subunit) pathway.

\section{Discussion}

In this study, we describe the kinetics of in vivo development of IL-10-secreting intestinal B cells and subsets of CD4 ${ }^{+} \mathrm{T}$ cells in ex-GF $I l 1 O^{+/ E G F P}$ reporter mice colonized with enteric resident microbiota and the importance of TLR2 and PI3K signaling in the induction of protective resident bacteria-induced IL-10-producing B cells.

The consortium of resident enteric bacteria provides protective signals to host immune defenses (40-42). Colonization of GF WT mice with resident fecal microbiota rapidly stimulated mucosal proinflammatory cytokine production manifested by transient release of TNF- $\alpha$, IL-12p40, and IFN- $\gamma$, but not IL-17a, within 3 days, while intestinal IL-10 levels gradually increased to maximum sustained levels in 7 days (43). The finding that transient in vivo stimulation of proinflammatory cytokine gene expression was followed by downregulation to basal levels in ex-GF $I l 1 O^{+/+}$but not in $I l 10^{-/-}$mice advances the hypothesis that resident microbiota induces IL-10 production by LP immune cells that mediates mucosal homeostasis. That neutralization of IL-10 signaling in B cells by anti-IL-10R antibody potentiated physiologic bacterial stimulation of IL-12p40 further supports our hypothesis. These results are consistent with our previous work showing that in vivo administration of anti-IL-10R antibody prevented downregulation of ex vivo bacterial antigenstimulated Th1 immune responses in ex-GF mice colonized with enteric bacteria (13).

Based on our current and previous observations, we propose that induction of IL-10 by enteric resident bacteria is essential for maintaining intestinal mucosal homeostasis. Our studies included several features that we believe to be novel: the use of GF $I l 10^{+/ E G F P}$ reporter (Vert-X) mice to study the in vivo kinetics of IL-10 activation after colonization with resident enteric bacteria; identification of the colonic LP cell types that produce IL-10 following microbial colonization; and the study of the signaling pathways of IL-10 induction by physiologic resident bacterial stimulation. Our use of $\mathrm{GFP}^{+}$cells as indicators of cellular IL-10 production was validated by the strong correlations we found between the frequency of $\mathrm{GFP}^{+}$cells and both IL-10 RNA transcription and IL-10 protein secretion following bacterial stimulation. Previous studies of systemic IL-10-secreting immune cells have relied on nonphysiologic, ex vivo stimulation of cultured immune cells (i.e., anti-CD40, anti-CD3/CD28, or concanavalin A [ConA]) or on mice with sep- 
tic, infectious, or autoimmune disease (32). Cells isolated from normal mice typically require prestimulation with nonspecific agents such as PMA/ionomycin to detect and analyze cell-specific IL-10 secretion by flow cytometry (44). Our physiologic approach using GF $I l 1 O^{+E G F P}$ reporter mice allowed us, without additional nonphysiologic stimulation, to more accurately detect and quantify the effects of complex intestinal microbiota lysates, specific bacterial species, and defined bacterial products on IL-10-producing cells in vitro; and the effects of in vivo physiologic colonization by complex resident microbiota on IL-10-producing cells in different mouse tissues over time.

Our somewhat unexpected observation that bacterial colonization of GF mice increased the frequency of colonic IL-10-producing $\mathrm{B}$ cells, in addition to subsets of $\mathrm{CD} 4^{+} \mathrm{T}$ cells, led us to investigate the roles of IL-10-producing B cells in maintaining mucosal homeostasis and the signaling pathways involved in IL-10 induction by resident microbiota (23-28). Our results and observations by others suggest that bacterial stimulation through the MyD88 pathway activates $I l 10$ expression and causes B cells to produce IL-10, which helps maintain gut homeostasis. B cellspecific $M y d 88^{-/-}$mice are more susceptible to dextran sulfate sodium-induced mucosal damage compared with mice lacking MyD88 in other cell types, including T cells, macrophages, DCs, and epithelium (45). In the present study, we demonstrated that ex vivo activation of the TLR2/MyD88 pathway is essential for induction of bacteria-activated mucosal IL-10-producing B cells, but not $\mathrm{T}$ cells. However, the absence of TLR signaling through MyD88 did not alter the relative number of mucosal IL-10-producing B cells in SPF-raised animals in vivo, indicating that physiologic development of intestinal IL-10-producing B cells is TLR independent, although in vivo protection against colitis by B cells is IL-10, TLR2, and MyD88 dependent. We postulate that basal activation of in vivo IL-10-producing $B$ cells and ex vivo bacteria-stimulated IL-10-secreting B cells likely requires different pathways in response to actively metabolizing resident intestinal bacteria, or that this activation depends on complex interactions with cell types not represented in our in vitro cultures. Similar disparities exist in the regulation of $\mathrm{IL}-10$-producing $\mathrm{CD} 4^{+}$cells, which increased in frequency following colonization of GF mice with SPF microbiota but are not activated ex vivo by physiologic cecal microbial lysates. Resolving these disparities will require further mechanistic studies.

We showed that upstream of MyD88 signaling, TLR2, but not TLR4, signaling increased the numbers of bacterially activated $\mathrm{GFP}^{+} \mathrm{B}$ cells and B cell IL-10 secretion, which contributed to B cell attenuation of T cell-mediated colitis. These TLR2 signaling findings represent what we believe to be a novel molecular mechanism of bacteria-stimulated, intestinal IL-10-producing B cells. Other investigators have shown that certain subsets of resident enteric bacteria induce regulatory immune responses, including IL-10 secretion, via TLR2 signaling $(9,46-49)$. Likewise, cecal luminal lysates and SPF bacteria appeared to preferentially activate mucosal IL-10-producing regulatory immune cells through TLR2. An important mechanistic observation from our study is that TLR2-mediated CBL stimulation of B cell production of IL-10 is mediated through activation of PI3K/AKT/GSK3 $\beta$ signaling. Furthermore, we demonstrated that WT but not PI3K signaling-defi- cient B cells attenuated colitis in a T and B cell cotransfer CD4 ${ }^{+}$ colitis model, specifically through the p110 $\delta$ subunit. Similarly, TLR2-dependent PI3K signaling has been reported to mediate phagocytosis of pathogens by macrophages, and PI3Kp110 $\delta$ regulates APC activation of Th1/Th17 cells and colitis $(39,50)$.

We demonstrated that in vivo microbial activation of colonic B cells and continued exposure to bacterial components was important for their homeostatic function. We observed that with ex vivo bacterial activation, GF mucosal B cells, compared with SPF mucosal B cells, produced equal amounts of IL-10, but higher levels of the inflammatory cytokine IL-12p40. These results show that in vivo exposure to resident microbiota enhances the regulatory capacity of intestinal B cells by selectively downregulating production of bacteria-stimulated inflammatory cytokines, but not IL-10. We previously demonstrated that with in vivo bacterial exposure, transferred GF WT B cells acquire regulatory phenotypes that help ameliorate colitis, with effects similar to those of B cells derived from SPF WT mice (23). In the current study, we showed that SPF B cells were unable to suppress effector $\mathrm{T}$ cell function in vitro without continued CBL exposure. These observations indicate that continuous bacterial stimulation is required in order to maintain regulatory B cells. Others have shown that bacterial colonization reduces the IL-10-mediated inflammatory hyporesponsiveness present in GF animals and makes them less susceptible to infection (40). We further demonstrated that in vivo physiologic intestinal microbial colonization selectively activated IL-10 production by subsets of intestinal LP T and B cells, with no concomitant stimulation of IL-10-producing cells in MLN and splenic cells in the same mice. These findings are consistent with the lack of differences in the number or function of IL-10-producing peritoneal regulatory B cells in GF versus SPF mice (28). The results emphasize the important site-specific interactions between the microbiota and intestinal regulatory B cells in mediating mucosal homeostasis.

We also explored the ability of the microbiota to induce $\mathrm{T}$ cell production of IL-10. Bacterial colonization of GF mice markedly increases intestinal $\mathrm{GFP}^{+} \mathrm{Tr} 1$ cells (Foxp ${ }^{\text {neg }} \mathrm{CD} 25^{-} \mathrm{CD} 4^{+} \mathrm{T}$ cells) and ROR $\gamma \mathrm{t}^{+} \mathrm{Foxp}^{+} \mathrm{GFP}^{+} \mathrm{CD} 4^{+} \mathrm{T}$ cells $(33,34,51)$, which made up only a small portion of the total number of $\mathrm{GFP}^{+} \mathrm{CD} 4^{+} \mathrm{T}$ cells. In contrast, ex vivo intestinal bacterial stimulation of immune cells predominantly increased $\mathrm{GFP}^{+} \mathrm{IL}^{-10}$-producing B cells but not $\mathrm{CD}^{+} \mathrm{T}$ cells. The lack of TLR/MyD88 signaling did not affect the frequency or activity of mucosal Tr1 cells either in vivo or ex vivo. These results suggest that resident enteric bacteria-stimulated $\operatorname{Tr} 1$ cell induction is TLR/MyD88 independent. Why different pathways are required for activation of IL-10-producing B and Tr1 cells is not entirely clear. CD4 $4^{+} \mathrm{IL}-10$-producing $\mathrm{T}$ cells may require physiologic stimulation by live bacterial antigens or metabolites, such as butyrate (52), rather than bacterial lysates; longer incubation periods with other signals not present ex vivo, or interactions with epithelial or mesenchymal cells. Cong and colleagues demonstrated that IL-10-secreting Tr1 cells from SPF mice were expanded by CBL antigen-pulsed APCs in vitro and that a CBLresponsive $\operatorname{Tr} 1$ clone could inhibit colitis in a $\mathrm{T}$ cell cotransfer model (53). We recently showed that $\operatorname{Tr} 1$ cells were induced by IL-10-producing B cells ex vivo in the presence of CBL when equal numbers of APCs and naive T cells were cocultured, a ratio 
considerably higher than the ratio of APCs to T cells in the intestinal LP (23). In these ex vivo coculture studies, B cells were the primary source of secreted IL-10. Our results suggest that ex vivo activation of IL-10 by T cells requires boosted antigen presentation by greater than physiologic numbers of APCs.

In summary, complex resident enteric microbiota selectively induced colonic LP IL-10-producing B cells and subsets of CD4 ${ }^{+}$ $\mathrm{T}$ cells, and increased the regulatory capacity of intestinal B cells. Fecal microbiota colonization of GF mice transiently activated inflammatory cytokines in the colon, which were subsequently downregulated by sustained IL-10 production. These inflammatory cytokines progressively increased from a higher baseline in IL-10-deficient mice, which develop colitis dependent on bacterial colonization. Resident bacteria stimulated IL-10 production through TLR2, MyD88, and PI3Kp110 $\delta$ signaling, which mediated B cell antiinflammatory responses that, in turn, attenuated T cell-mediated colitis. We conclude that resident enteric bacteria activate IL-10-producing intestinal B cells in a MyD88/TLR2/ PI3K-dependent manner that suppresses aggressive immune responses and maintains mucosal homeostasis.

\section{Methods}

Further information can be found in Supplemental Methods.

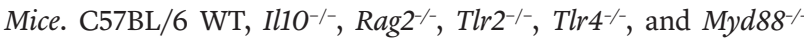
mice were purchased from the Jackson Laboratory. $I l 10^{+/ E G F P}$ reporter (Vert-X) mice have been described previously (32). Pi3kp1108 ${ }^{\text {D910A/D910A }}$ $\left(\mathrm{PI} 3 \mathrm{Kp} 110 \delta^{\mathrm{KI}}\right.$ ) mice were previously obtained from B. Vanhaesebroeck (Queen Mary University of London, London, United Kingdom) (39). IllO ${ }^{-/-}$Rag2 $2^{-/-}$double-knockout (DKO) mice were generated by crossing $\mathrm{IllO}^{-/-}$with Rag2 $^{-/-}$mice. Tlr2 $2^{-/-} \mathrm{Il1O}^{+/ E G F P}, \mathrm{Tlr}^{-/-} \mathrm{Il1O^{+/EGFP }}$, and $\mathrm{Myd} d 88^{-/-}$ IllO ${ }^{+/ E G F P}$ mice were generated by crossing $T l r 2^{-/-}$with $I l 1 O^{+/ E G F P}$ mice, $\mathrm{Tlr} 4^{-/-}$with $I l 1 \mathrm{O}^{+/ E G F P}$ mice, and $\mathrm{Myd} d 88^{-/-}$with $\mathrm{IllO} \mathrm{O}^{+/ E G F P}$ mice, respectively. These mice were maintained in SPF conditions at UNC and were used at 8-12 weeks of age. $I l 1 O^{+/ E G F P}$, WT, and $I l 10^{-/-}$mice were derived into GF conditions by embryo transfer and maintained in the UNC Center for Gastrointestinal Biology and Disease Gnotobiotic Core. In some experiments, GF mice were inoculated with feces from SPF WT mice.

Cell isolation. Mononuclear cells were isolated from colonic LP, MLN, and spleen as described previously (24). MLNs were crushed through $70-\mu \mathrm{m}$ filters into PBS with $2.5 \%$ FBS (Gibco, Invitrogen). Spleens were mechanically dissociated, and red blood cells were lysed with Red Blood Cell Lysing Buffer (Sigma-Aldrich). For isolation of colonic LP lymphocytes (LPLs), colons were washed with cold PBS, opened longitudinally, and cut into $10-\mathrm{mm}$ pieces. Intestinal fragments were incubated in $1 \mathrm{mM}$ DTT (Sigma-Aldrich) in $\mathrm{Ca}^{2+}$ - and $\mathrm{Mg}^{2+}$-free HBSS (Gibco, Invitrogen) for 15 minutes at room temperature. Next, the tissues were incubated in $1 \mathrm{mM}$ EDTA (Sigma-Aldrich) in HBSS for 20 minutes at $37^{\circ} \mathrm{C}$ with shaking, which was repeated after a thorough washing. The cell suspensions were removed, and remaining fragments were transferred to flasks containing HBSS with $1 \mathrm{mg} / \mathrm{mL}$ collagenase type III (Sigma-Aldrich) and 1\% penicillin-streptomycin (Gibco, Invitrogen), then stirred gently for $60 \mathrm{~min}$ utes at $37^{\circ} \mathrm{C}$. Cell suspensions containing LPLs were filtered through a nylon mesh and centrifuged; then the LPLs were purified using a $44 \% / 70 \%$ discontinuous Percoll gradient (GE Healthcare). After centrifugation at $800 \mathrm{~g}$ for 20 minutes at $22^{\circ} \mathrm{C}$, mononuclear cells were collected from the interface.
Cell purification. MLN or colon LP B cells were purified magnetically by positive selection with anti-CD19 microbeads after negative selection using a mixture of anti-CD90.2, anti-CD11c, and antiTer119 microbeads. Final CD19+ cell fractions were confirmed to be greater than $99.5 \%$ pure by flow cytometry, while cell viability was shown to be greater than $88 \%$ by eosin $\mathrm{Y}$ exclusion. In some experiments, colon LP cells were separated into B cell and non-B cell populations with anti-CD19 MicroBeads. Naive $\mathrm{CD} 4^{+} \mathrm{T}$ cells were isolated by use of a Naive $\mathrm{CD} 4^{+} \mathrm{T}$ Cell Isolation Kit, Mouse, and confirmed to be greater than $94.7 \%$ pure and $95 \%$ viable. All microbeads including the naive $\mathrm{CD} 4^{+} \mathrm{T}$ cell isolation kit used in this study were purchased from Miltenyi Biotec.

Bacterial lysate. CBL was prepared from the contents of ceca from SPF-raised C57BL/6 WT mice as described previously (21). Lysates of each bacteria strain were prepared from individual colonies of $E$. coli LF82, E. faecalis, and R.gnavus, as previously described (36). A lysate of Clostridia was prepared from the cecal contents of ex-GF mice selectively colonized with a mixture of 17 strains of Clostridium species (8).

Cell cultures. Unfractionated MLN cells $\left(1 \times 10^{6}\right)$ or colon LP cells isolated from GF or SPF-raised Il1O ${ }^{+/ E G F P}$ mice were cultured in $200 \mu \mathrm{l}$ medium/well (96-well plates) for $24-72$ hours at $37^{\circ} \mathrm{C}$ with $5 \% \mathrm{CO}_{2}$. The culture medium was RPMI 1640 (Gibco, Invitrogen) containing $10 \%$ FBS, $1 \%$ penicillin-streptomycin-amphotericin B (Gibco, Invitrogen), and $5 \times 10^{-5} \mathrm{~mol} / \mathrm{L} 2$-mercaptoethanol (Sigma-Aldrich) with or without the following bacterial products; 10,50 , or $100 \mu \mathrm{g} / \mathrm{mL} \mathrm{CBL}$; $10 \mu \mathrm{g} / \mathrm{mL}$ lysate of Clostridia, E. coli LF82, E. faecalis, or R. gnavus; 200 $\mathrm{ng} / \mathrm{mL}$ LPS, and $50 \mathrm{ng} / \mathrm{mL}$ Pam3CSK4 (InvivoGen) or $1 \mathrm{nM} \mathrm{CpG-DNA}$ (Sigma-Aldrich). In some experiments, $1 \mu \mathrm{M}$ of the pan-PI3K inhibitor wortmannin (Sigma-Aldrich) or Ly294002 (Sigma-Aldrich); $1 \mu \mathrm{M}$ of the PI3Kp110 $\delta$-specific inhibitor IC-87114 (Sigma-Aldrich); or vehicle (DMSO) alone was added at various concentrations indicated in the Figure legends. Following cell culture, the supernatants were collected for measurements of cytokines by ELISA, while cells were analyzed by flow cytometry.

Cytokine measurements. IFN- $\gamma$, IL-12/23p40, or IL-10 ELISAs were performed by the Immunoassay Core of the UNC Center for Gastrointestinal Biology and Disease using the following products: mouse anti-IL-10, -IL-12/23p40, and -IFN- $\gamma$ (BD Biosciences) as previously described $(21,23)$. Concentrations of cytokines were established in triplicate culture supernatants by comparison with standard curves generated using the appropriate recombinant cytokine per the manufacturer's instructions.

Real-time PCR. Total RNA was isolated from distal colon using the RNeasy Micro Kit (QIAGEN). First-strand complementary DNA was synthesized from $1 \mu \mathrm{g}$ total RNA using M-MLV Reverse Transcriptase (Gibco, Invitrogen) according to the manufacturer's instructions. Quantitative reverse transcription PCR was performed on a Mastercycler ep realplex 2S (Eppendorf) using the SYBR Green quantitative PCR SuperMix (Invitrogen) to quantify gene expression. The PCR primers used in this study were reported previously $(21,23,54)$. Each complementary DNA sample was analyzed in duplicate or triplicate for quantitative assessment of RNA amplification, and the results are expressed relative to the housekeeping gene $\beta$-actin.

Immunohistochemistry. Distal colons were harvested, fixed with $4 \%$ paraformaldehyde (Sigma-Aldrich), and embedded in O.C.T. compound (Sakura). Tissue sections $(5 \mu \mathrm{m})$ were blocked with TNB buffer (PerkinElmer) for 30 minutes at room temperature and incu- 
bated overnight at $4^{\circ} \mathrm{C}$ with rabbit anti-GFP antibody (Invitrogen) and rat anti-mouse $\mathrm{B} 220$ or Armenian hamster anti-mouse CD3 antibody (BD Biosciences). Rabbit IgG (Invitrogen) was used as a negative control for rabbit anti-GFP antibody. Primary antibodies are listed in Supplemental Table 1. After washing, tissue sections were treated with DyLight 488-conjugated donkey anti-rabbit IgG and HRP-conjugated donkey anti-rat IgG or HRP-conjugated donkey anti-hamster IgG (Jackson ImmunoResearch Laboratories Inc.). The sections were finally incubated with TSA Plus Cyanine 5 amplification reagent (PerkinElmer) for 10 minutes at room temperature and counterstained with DAPI (Sigma-Aldrich). All sections were analyzed by confocal microscopy (TCS SP2, Leica).

Western blots. Western blots were performed as previously reported (55). One centimeter of distal colons was homogenized in 1\% RIPA lysis buffer (Boston Bio Products) containing cOmplete Protease Inhibitor Tablet (Roche) and PhosSTOP Phosphatase Inhibitor Tablet (Roche) using a Pro200 tissue homogenizer (PRO Scientific). Lysates were incubated on ice for an additional 10 minutes at $4^{\circ} \mathrm{C}$ with rotation. The lysates were cleared of cellular debris by centrifugation, and total protein concentration was determined by BCA assay (Thermo Fisher Scientific). A total of $30 \mu \mathrm{g}$ protein from each sample was separated by SDS-PAGE using 4\%-12\% NuPAGE Bis-Tris gels (Invitrogen). The separated proteins were transferred onto $0.2-\mu \mathrm{m}$ nitrocellulose membranes and were blocked for 1 hour at room temperature with TBS-T (10 mM Tris, $7.5 \mathrm{pH}, 150 \mathrm{mM} \mathrm{NaCl}$, and 0.1\% Tween-20) containing $10 \%$ nonfat dry milk. The membranes were incubated overnight with anti-phospho-STAT3 (Tyr705; Cell Signaling Technology) and then washed 5 times with TBS-T and incubated for 2 hours at room temperature with HRP-conjugated goat anti-rabbit IgG secondary antibodies (Sigma-Aldrich) (1:40,000 dilution). The membranes were developed using SuperSignal West Chemiluminescent Substrate (Thermo Fisher Scientific) and were visualized by exposure to blue sensitive X-ray film (Genesee Scientific). To confirm even loading, the antibodies were removed from the membranes using Restore PLUS Western Blot Stripping buffer (Thermo Fisher Scientific), and membranes were blocked as previously described (55) and incubated with anti-STAT3 (Cell Signaling Technology) or anti-actin-HRP (Santa Cruz Biotechnology) (1:10,000 dilution), followed by HRP-conjugated goat anti-rabbit IgG secondary antibodies when appropriate.

Following in vitro stimulation of primary B cells with CBL, cells were washed with PBS at $4^{\circ} \mathrm{C}$, pelleted by centrifugation at $400 \mathrm{~g}$ for 5 minutes at $4^{\circ} \mathrm{C}$, and lysed in 1\% RIPA buffer containing Complete Protease Inhibitor Tablet and PhosSTOP Phosphatase Inhibitor Tablet for 10 minutes on ice. Protein lysates were cleared of cellular debris by centrifugation, and the lysates were subjected to SDS-PAGE and Western blot as described above in Western blots using anti-phospho-Akt (Ser473) antibodies (Cell Signaling Technology; 1:1,000 dilution), followed by HRP-conjugated goat-anti-rabbit IgG secondary antibodies (Sigma-Aldrich) (1:40,000 dilution). Antibodies were removed from the membranes as described above, and even protein loading was confirmed using anti-Akt antibodies (Cell Signaling Technology; 1:1,000 dilution), followed by HRP-conjugated goat anti-rabbit IgG secondary antibodies (Sigma-Aldrich; 1:40,000 dilution).

Primary antibodies are listed in Supplemental Table 1. All antibodies were diluted in TBS-T containing $5 \%$ nonfat dry milk. Densitometric analysis was performed for all Western blots using Image J software (version 1.49; NIH).
Flow cytometry. Spleen, MLN, and colonic LP cells were incubated for 15 minutes at $4^{\circ} \mathrm{C}$ with anti-CD16/CD32 (BD Biosciences) to block Fc receptors and then for 20 minutes at $4^{\circ} \mathrm{C}$ with $6-7$ fluorochrome-conjugated antibodies (Supplemental Table 1) to evaluate the cell phenotype. All sample cells were stained with LIVE/DEAD Fixable Dead Cell Stain Kit (Near-IR, Thermo Fisher Scientific) and Pacific Orange-conjugated CD45 along with the following: PE, PE-Cy5, PE-Cy7, Pacific blue, allophycocyanin, or equivalent fluorescence-conjugated antibodies; B220 and CD19 for B cells; CD3 and CD4 for CD4 ${ }^{+} \mathrm{T}$ cells; CD3 and CD8 for CD8 ${ }^{+}$ T cells; CD11b and CD11c for DCs; CD11b and F4/80 for macrophages; or CD3 and CD49b for NK cells. In addition, target antibodies or isotype control antibodies were simultaneously added. Enumeration of cells expressing Foxp3 or ROR $\gamma \mathrm{t}$ was performed by intracellular staining with the Fixation/Permeabilization Solution Kit according to the manufacturer's instructions (BD Biosciences) or fixation buffer (PBS containing 4\% paraformaldehyde; Electron Microscopy Sciences), 0.01\% Tween-20 (Thermo Fisher Scientific), permeabilization buffer (PBS containing 0.1\% Triton X-100; MP Biomedicals), 0.5\% BSA (Sigma-Aldrich), and 2 mM EDTA. Cells were washed and then analyzed on a CyAn ADP flow cytometer (Beckman Coulter) or LSR II (BD Biosciences) at the UNC Flow Cytometry Core Facility. Cells from SPF-raised WT C57BL/6 (GFP-) mice were used to determine GFP basal intensity and set the GFP gate when $I l 10^{+/ E G F P}$ reporter cells were used. These WT cells were stained with the same antibodies as target cells except for GFP, as a fluorescence minus one (FMO) control to assure that spillover or spread from other fluorophores would not contribute to the signal in the GFP detector. Suitable positive control staining samples or IL-10 reporter cells were used for compensation. Seven parameters, including GFP channel, were used for running flow cytometry. Gated live CD $45^{+}$cells were analyzed with Summit 5.2 software (Beckman Coulter) or FlowJo version 10.3.

Transfer of $C D 4^{+} T$ and $B$ cells. DKO mice received i.p. injections of $5 \times 10^{5}$ splenic $\mathrm{CD} 4^{+}$cells with or without $1 \times 10^{6}$ splenic B cells from

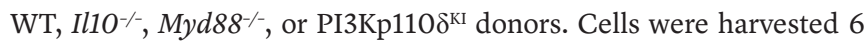
weeks after cell transfer, according to Association for Assessment of Laboratory Animal Care-approved (AALAC-approved) methods.

Evaluation of colitis. Colitis severity was evaluated by histopathology scoring and cytokine ELISAs on culture media from colonic tissue explants. For histology, intestinal tissues were removed at necropsy and fixed in $10 \%$ buffered formalin. Paraffin sections were prepared and stained with $\mathrm{H} \& \mathrm{E}$ for assessment of colonic inflammation by the Histology Core of the UNC Center for Gastrointestinal Biology and Disease. Scoring of mucosal inflammation in cecum, proximal colon, and distal colon was performed in a blinded fashion, with each region being graded from 0 to 4 as described previously (56). Total histology scores (maximum score of 12) represent the summation of the scores from the 3 regions. For colonic tissue explant assays, cultures of colon fragments from the recipient mice were prepared as described previously (21).

Blockade of TLR2 signaling in vivo. Anti-TLR2 antibody (BioLegend; $100 \mu \mathrm{g} / \mathrm{mouse}$ ) or isotype control antibody (purified mouse IgG1, $\kappa$; BioLegend) was injected i.p. on days 0 and 4 after fecal transplantation with SPF feces. Mice were harvested on day 7, and we evaluated colonic $\mathrm{GFP}^{+} \mathrm{B}$ cells by flow cytometry, as well as mRNA expression of Il1O in distal colon. 
Statistics. We used Prism 6 software (GraphPad) to compare medians between 2 groups with the Mann-Whitney $U$ test; comparisons of means from multiple groups were analyzed with the Kruskal-Wallis test with Dunn's post hoc test. $P$ values less than 0.05 were considered significant.

Study approval. These studies were reviewed and approved by the UNC IACUC, protocol 12-300.0.

\section{Author contributions}

YM and RBS conceptualized and designed the study; acquired, analyzed, and interpreted data; performed statistical analyses; and wrote the manuscript. AO, BL, JWH, CSE, TJF, EBS, IMC, LC, JEW, NCF, TN, AW, and JVG acquired, analyzed, and interpreted data. JJH and JPYT drafted the manuscript and provided administrative assistance. CLK provided reporter mice and technical/conceptual advice.

\section{Acknowledgments}

These studies were supported by NIH grants P01 DK094779, R01 DK53347, P40 OD010995, and P30 DK34987 (to RBS); R01 CA156330 (to JPYT); R01 AI123010 (to AW); and R01 AI111899, AI140799, and MH108179 (to JVG); and by Crohn's and Colitis Foundation Microbiome Initiative and Gnotobiotic Facility grants (both to RBS) and Research Fellowship Award (to YM and AO).
JEW was supported by a postdoctoral fellowship from the American Cancer Society (PF-13-401-01-TBE). The UNC Flow Cytometry Core is supported in part by Cancer Center Core Support Grant P30 CA016086 to the UNC Lineberger Comprehensive Cancer Center. The content is solely the responsibility of the authors and does not necessarily represent the official views of the NIH. The views expressed herein do not necessarily represent the views of the Bill \& Melinda Gates Foundation. The authors acknowledge the editorial assistance of Richard Youngblood of the UNC Translational and Clinical Sciences Institute.

Address correspondence to: R. Balfour Sartor, Room 7309A MBRB, CB\# 7032, Center for Gastrointestinal Biology and Disease, Department of Medicine, Division of Gastroenterology and Hepatology, University of North Carolina, Chapel Hill, North Carolina, 27599-7032, USA. Phone: 919.966.0149; Email: ryan_ balfour_sartor@med.unc.edu.

CLK's present address is: Global Health Discovery \& Translational Sciences and Maternal, Neonatal and Child Health Discovery and Tools, The Bill \& Melinda Gates Foundation, Seattle, Washington, USA.
1. Harris KG, Chang EB. The intestinal microbiota in the pathogenesis of inflammatory bowel diseases: new insights into complex disease. Clin Sci. 2018;132(18):2013-2028.

2. Kostic AD, Xavier RJ, Gevers D. The microbiome in inflammatory bowel disease: current status and the future ahead. Gastroenterology. 2014;146(6):1489-1499.

3. Sartor RB, Wu GD. Roles for intestinal bacteria, viruses, and fungi in pathogenesis of inflammatory bowel diseases and therapeutic approaches. Gastroenterology. 2017;152(2):327-339.e4.

4. Berg DJ, et al. Enterocolitis and colon cancer in interleukin-10-deficient mice are associated with aberrant cytokine production and CD4(+) TH1-like responses. JClin Invest. 1996;98(4):1010-1020.

5. Sellon RK, et al. Resident enteric bacteria are necessary for development of spontaneous colitis and immune system activation in interleukin-10-deficient mice. Infect Immun. 1998;66(11):5224-5231.

6. Rath HC, et al. Normal luminal bacteria, especially Bacteroides species, mediate chronic colitis, gastritis, and arthritis in HLA-B27/human beta 2 microglobulin transgenic rats. JClin Invest. 1996;98(4):945-953.

7. Ivanov II, et al. Induction of intestinal Th17 cells by segmented filamentous bacteria. Cell. 2009;139(3):485-498.

8. Atarashi $\mathrm{K}$, et al. Treg induction by a rationally selected mixture of Clostridia strains from the human microbiota. Nature. 2013;500(7461):232-236.

9. Round JL, et al. The Toll-like receptor 2 pathway establishes colonization by a commensal of the human microbiota. Science. 2011;332(6032):974-977.

10. Yen D, et al. IL-23 is essential for T cell-mediated colitis and promotes inflammation via IL-17 and IL-6. J Clin Invest. 2006;116(5):1310-1316.
11. Steidler L, et al. Treatment of murine colitis by Lactococcus lactis secreting interleukin-10. Science. 2000;289(5483):1352-1355.

12. Herfarth HH, Mohanty SP, Rath HC, Tonkonogy $\mathrm{S}$, Sartor RB. Interleukin 10 suppresses experimental chronic, granulomatous inflammation induced by bacterial cell wall polymers. Gut. 1996;39(6):836-845.

13. Wu C, Sartor RB, Huang K, Tonkonogy SL. Transient activation of mucosal effector immune responses by resident intestinal bacteria in normal hosts is regulated by interleukin-10 signalling. Immunology. 2016;148(3):304-314.

14. Franke A, et al. Sequence variants in IL10, ARPC2 and multiple other loci contribute to ulcerative colitis susceptibility. Nat Genet. 2008;40(11):1319-1323.

15. Amre DK, et al. Interleukin 10 (IL-10) gene variants and susceptibility for paediatric onset Crohn's disease. Aliment Pharmacol Ther. 2009;29(9):1025-1031.

16. Glocker EO, et al. Inflammatory bowel disease and mutations affecting the interleukin-10 receptor. N Engl J Med. 2009;361(21):2033-2045.

17. Sabat R, et al. Biology of interleukin-10. Cytokine Growth Factor Rev. 2010;21(5):331-344.

18. Roers A, et al. T cell-specific inactivation of the interleukin 10 gene in mice results in enhanced $\mathrm{T}$ cell responses but normal innate responses to lipopolysaccharide or skin irritation. J Exp Med. 2004;200(10):1289-1297.

19. Groux H, Powrie F. Regulatory T cells and inflammatory bowel disease. Immunol Today. 1999;20(10):442-445.

20. Brockmann L, et al. Molecular and functional heterogeneity of IL-10-producing CD4. Nat Commun. 2018;9(1):5457.

21. Liu B, Tonkonogy SL, Sartor RB. Antigen-presenting cell production of IL-10 inhibits T-helper 1 and 17 cell responses and suppresses colitis in mice.
Gastroenterology. 2011;141(2):653-662, 662.e1.

22. Shouval DS, et al. Interleukin-10 receptor signaling in innate immune cells regulates mucosal immune tolerance and anti-inflammatory macrophage function. Immunity. 2014;40(5):706-719.

23. Mishima Y, Liu B, Hansen JJ, Sartor RB. Resident bacteria-stimulated IL-10-secreting B cells ameliorate T cell-mediated colitis by inducing Tr-1 cells that require IL-27-signaling. Cell Mol Gastroenterol Hepatol. 2015;1(3):295-310.

24. Mishima Y, et al. Decreased production of interleukin-10 and transforming growth factor- $\beta$ in Toll-like receptor-activated intestinal B cells in SAMP1/Yit mice. Immunology. 2010;131(4):473-487.

25. El Fassi D, Nielsen CH, Kjeldsen J, Clemmensen $\mathrm{O}$, Hegedüs L. Ulcerative colitis following B lymphocyte depletion with rituximab in a patient with Graves' disease. Gut. 2008;57(5):714-715.

26. Goetz M, Atreya R, Ghalibafian M, Galle PR, Neurath MF. Exacerbation of ulcerative colitis after rituximab salvage therapy. Inflamm Bowel Dis. 2007;13(11):1365-1368.

27. Mizoguchi A, Mizoguchi E, Takedatsu H, Blumberg RS, Bhan AK. Chronic intestinal inflammatory condition generates IL-10-producing regulatory $B$ cell subset characterized by CD1d upregulation. Immunity. 2002;16(2):219-230.

28. Maseda D, et al. Peritoneal cavity regulatory $B$ cells (B10 cells) modulate IFN $-\gamma+\mathrm{CD} 4+\mathrm{T}$ cell numbers during colitis development in mice. Jimmunol. 2013;191(5):2780-2795.

29. Yanaba K, Bouaziz JD, Matsushita T, Tsubata T, Tedder TF. The development and function of regulatory B cells expressing IL-10 (B10 cells) requires antigen receptor diversity and TLR signals. J Immunol. 2009;182(12):7459-7472.

30. Rosser EC, et al. Regulatory B cells are induced by gut microbiota-driven interleukin- $1 \beta$ and interleukin- 6 production. Nat Med. 2014;20(11):1334-1339. 
31. Saraiva M, O'Garra A. The regulation of IL-10 production by immune cells. Nat Rev Immunol. 2010;10(3):170-181.

32. Madan R, et al. Nonredundant roles for B cellderived IL-10 in immune counter-regulation. J Immunol. 2009;183(4):2312-2320.

33. Lochner $M$, et al. In vivo equilibrium of proinflammatory IL-17+ and regulatory IL-10+ Foxp3+ RORgamma t+ T cells. J Exp Med . 2008;205(6):1381-1393.

34. Yang BH, et al. Foxp3(+) T cells expressing ROR $\gamma \mathrm{t}$ represent a stable regulatory $\mathrm{T}$-cell effector lineage with enhanced suppressive capacity during intestinal inflammation. Mucosal Immunol. 2016;9(2):444-457.

35. Mauri C, Menon M. The expanding family of regulatory B cells. Int Immunol. 2015;27(10):479-486.

36. Eun CS, et al. Induction of bacterial antigen-specific colitis by a simplified human microbiota consortium in gnotobiotic interleukin-10-/mice. Infect Immun. 2014;82(6):2239-2246.

37. Gevers D et al. The treatment-naive microbiome in new-onset Crohn's disease. Cell Host Microbe. 2014;15(3):382-392.

38. Martin M, Schifferle RE, Cuesta N, Vogel SN, Katz J, Michalek SM. Role of the phosphatidylinositol 3 kinase-Akt pathway in the regulation of IL-10 and IL-12 by Porphyromonas gingivalis lipopolysaccharide. JImmunol. 2003;171(2):717-725.

39. Steinbach EC, et al. Innate PI3K p110 $\delta$ regulates Th1/Th17 development and microbiota-dependent colitis. J Immunol. 2014;192(8):3958-3968. 40. Fagundes CT, et al. Transient TLR activation restores inflammatory response and ability to control pulmonary bacterial infection in germfree mice. JImmunol. 2012;188(3):1411-1420.

41. Kitajima S, Morimoto M, Sagara E, Shimizu C, Ikeda Y. Dextran sodium sulfate-induced colitis in germ-free IQI/Jic mice. Exp Anim. 2001;50(5):387-395.

42. Hegazy AN, et al. Circulating and tissue-resident CD4. Gastroenterology. 2017;153(5):1320-1337.e16.

43. Sydora BC, MacFarlane SM, Lupicki M, Dmytrash AL, Dieleman LA, Fedorak RN. An imbalance in mucosal cytokine profile causes transient intestinal inflammation following an animal's first exposure to faecal bacteria and antigens. Clin Exp Immunol. 2010;161(1):187-196.

44. Muris AH, Damoiseaux J, Smolders J, Cohen Tervaert JW, Hupperts R, Thewissen M. Intracellular IL-10 detection in T cells by flowcytometry: the use of protein transport inhibitors revisited. J Immunol Methods. 2012;381(1-2):59-65.

45. Kirkland D, et al. B cell-intrinsic MyD88 signaling prevents the lethal dissemination of commensal bacteria during colonic damage. Immunity. 2012;36(2):228-238.

46. Jeon SG, et al. Probiotic Bifidobacterium breve induces IL-10-producing Tr1 cells in the colon. PLoS Pathog. 2012;8(5):e1002714.

47. Sayi A, et al. TLR-2-activated B cells suppress Helicobacter-induced preneoplastic gastric immunopathology by inducing T regulatory-1 cells. J Immunol. 2011;186(2):878-890.

48. Liang Y, et al. Toll-like receptor 2 induces mucosal homing receptor expression and $\operatorname{IgA}$ production by human B cells. Clin Immunol. 2011;138(1):33-40.

49. Mohamadzadeh M, et al. Regulation of induced colonic inflammation by Lactobacillus acidophilus deficient in lipoteichoic acid. Proc Natl Acad Sci U S A. 2011;108(suppl 1):4623-4630.

50. Shen Y, et al. Toll-like receptor 2- and MyD88dependent phosphatidylinositol 3-kinase and Rac1 activation facilitates the phagocytosis of Listeria monocytogenes by murine macrophages. Infect Immun. 2010;78(6):2857-2867.

51. Britton GJ, et al. Microbiotas from humans with inflammatory bowel disease alter the balance of Gut Th17 and ROR $\gamma \mathrm{t}$. Immunity. 2019;50(1):212-224.e4.

52. Furusawa Y, et al. Commensal microbe-derived butyrate induces the differentiation of colonic regulatory T cells. Nature. 2013;504(7480):446-450.

53. Cong Y, Weaver CT, Lazenby A, Elson CO. Bacterial-reactive $\mathrm{T}$ regulatory cells inhibit pathogenic immune responses to the enteric flora. J Immunol. 2002;169(11):6112-6119.

54. Liu B, et al. Irgm1-deficient mice exhibit Paneth cell abnormalities and increased susceptibility to acute intestinal inflammation. Am J Physiol Gastrointest Liver Physiol. 2013;305(8):G573-G584.

55. Wilson JE, et al. Inflammasome-independent role of AIM2 in suppressing colon tumorigenesis via DNA-PK and Akt. Nat Med. 2015;21(8):906-913.

56. Kim SC, et al. Variable phenotypes of enterocolitis in interleukin 10-deficient mice monoassociated with two different commensal bacteria. Gastroenterology. 2005;128(4):891-906. 